\title{
Faster Real Feasibility via Circuit Discriminants
}

\author{
Frederic Bihan ${ }^{*}$ \\ UFR SFA, Campus \\ Scientifique \\ 73376 Le Bourget-du-Lac \\ Cedex \\ France \\ Frederic.Bihan@univ- \\ savoie.fr
}

\author{
J. Maurice Rojas ${ }^{\dagger}$ \\ TAMU 3368 \\ Department of Mathematics \\ Texas A\&M University \\ College Station, Texas \\ 77843-3368 \\ USA \\ rojas@math.tamu.edu
}

\author{
Casey E. Stella \\ 18409 Newell Road \\ Shaker Heights, $\mathrm{OH} 44122$ \\ USA \\ cestella@gmail.com
}

Rojas dedicates this paper to the memory of his dear friend, Richard Adolph Snavely, 1955-2005.

\begin{abstract}
We show that detecting real roots for honestly $n$-variate $(n+2)$-nomials (with integer exponents and coefficients) can be done in time polynomial in the sparse encoding for any fixed $n$. The best previous complexity bounds were exponential in the sparse encoding, even for $n$ fixed. We then give a characterization of those functions $k(n)$ such that the complexity of detecting real roots for $n$-variate $(n+k(n))$-nomials transitions from $\mathbf{P}$ to $\mathbf{N P}$-hardness as $n \longrightarrow \infty$. Our proofs follow in large part from a new complexity threshold for deciding the vanishing of $\mathcal{A}$-discriminants of $n$-variate $(n+k(n))$-nomials. Diophantine approximation, through linear forms in logarithms, also arises as a key tool.
\end{abstract}

\section{Keywords}

sparse, real, feasibility, polynomial-time, discriminant chamber

\section{INTRODUCTION AND MAIN RESULTS}

Consider real feasibility: the problem of deciding the existence of real roots for systems of polynomial equations. In addition to having numerous practical applications (see, e.g., [BG-V03]), real feasibility is an important motivation behind effectivity estimates for the Real Nullstellensatz (e.g., Ste74 Sch00]), the quantitative study of sums of squares Ble04,

\footnotetext{
*Member of the European Research Training Network RAAG CT 2001-00271.

†Partially supported by NSF individual grant DMS-0211458, NSF CAREER grant DMS-0349309, Sandia National Laboratories, and the American Institute of Mathematics.

${ }^{\ddagger}$ Partially supported by NSF grant DMS-0211458.
}

Permission to make digital or hard copies of all or part of this work for personal or classroom use is granted without fee provided that copies are not made or distributed for profit or commercial advantage and that copies bear this notice and the full citation on the first page. To copy otherwise, to republish, to post on servers or to redistribute to lists, requires prior specific permission and/or a fee.

ISSAC '09 Seoul, Korea

Copyright 200X ACM X-XXXXX-XX-X/XX/XX ...\$5.00.
RS09, BHPR09, and their connection to semi-definite programming and optimization [Par03, Las07]. In particular, real solving of sparse polynomial systems arises in concrete applications such as satellite orbit mechanics AM09, and real solving clearly involves real feasibility as an initial step. We are thus inspired to derive new algorithms and complexity lower bounds for real feasibility, in the refined setting of sparse polynomials.

To state our results, let us first clarify some basic notation concerning sparse polynomials and some well-known complexity classes. Recall that $R^{*}$ is the multiplicative group of nonzero elements in any ring $R$.

Definition 1.1. When $a_{j} \in \mathbb{R}^{n}$, the notations $a_{j}=$ $\left(a_{1, j}, \ldots, a_{n, j}\right), x^{a_{j}}=x_{1}^{a_{1, j}} \cdots x_{n}^{a_{n, j}}$, and $x=\left(x_{1}, \ldots, x_{n}\right)$ will be understood. If $f(x):=\sum_{j=1}^{m} c_{i} x^{a_{j}}$ where $c_{j} \in \mathbb{R}^{*}$ for all $j$, and the $a_{j}$ are pair-wise distinct, then we call $f$ a (real) $\boldsymbol{n}$-variate $\boldsymbol{m}$-nomial, and we define $\operatorname{Supp}(f):=$ $\left\{a_{1}, \ldots, a_{m}\right\}$ to be the support of $f$. We also let $\mathcal{F}_{n, m}$ denote the set of all $n$-variate $\lfloor m\rfloor$-nomials within $\mathbb{Z}\left[x_{1}, \ldots, x_{n}\right]$. Finally, for any $m \geq n+1$, we let $\mathcal{F}_{n, m}^{*} \subseteq \mathcal{F}_{n, m}$ denote the subset consisting of those $f$ with $\operatorname{Supp}(f)$ not contained in any $(n-1)$-flat. We also call any $f \in \mathcal{F}_{n, m}^{*}$ an honest $\boldsymbol{n}$-variate $\boldsymbol{m}$-nomial (or honestly $\boldsymbol{n}$-variate). $\diamond$

For example, $1+7 x_{1}^{2} x_{2} x_{3}^{7} x_{4}^{3}-43 x_{1}^{198} x_{2}^{99} x_{3}^{693} x_{4}^{297}$ is a 4 -variate trinomial with support contained in a line segment, but it has a real root $x \in \mathbb{R}^{4}$ iff the honestly univariate trinomial $1+7 y_{1}-43 y_{1}^{99}$ has a real root $y_{1} \in \mathbb{R}$. More generally (via Lemma 2.5 of Section 2.2 below), it will be natural to restrict to $\mathcal{F}_{n, n+k}^{*}$ (with $k \geq 1$ ) to study the role of sparsity in algorithmic complexity over the real numbers.

We will work with some well-known complexity classes from the classical Turing model, briefly reviewed in the Appendix. (A more complete introduction can be found in Pap95.) In particular, our underlying notion of input size is clarified in Definition 2.1 of Section 2.1 below, and illustrated in Example 1.4 immediately following our first main theorem. So for now, let us just recall the basic inclusions $\mathbf{N C}^{1} \subseteq \mathbf{P} \subseteq \mathbf{N P} \subseteq \mathbf{P S P A C E}$. While it is known that $\mathbf{N C}^{1} \neq \mathbf{P S P A C E}$ the properness of each of the remaining inclusions above is a famous open problem.

\subsection{Sparse Real Feasibility and $\mathcal{A}$-Discriminant Complexity}

DeFinition 1.2. Let $\mathbb{R}_{+}$denote the positive real num-

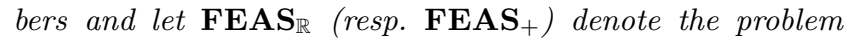


of deciding whether an arbitrary system of equations from $\bigcup_{n \in \mathbb{N}} \mathbb{Z}\left[x_{1}, \ldots, x_{n}\right]$ has a real root (resp. a root with all coordinates positive). Also, for any collection $\mathcal{F}$ of tuples chosen from $\bigcup_{k, n \in \mathbb{N}}\left(\mathbb{Z}\left[x_{1}, \ldots, x_{n}\right]\right)^{k}$, we let $\mathbf{F E A} \mathbf{S}_{\mathbb{R}}(\mathcal{F})$ (resp. $\mathbf{F E A S} \mathbf{S}_{+}(\mathcal{F})$ ) denote the natural restriction of $\mathbf{F E A} \mathbf{S}_{\mathbb{R}}$ (resp. FEAS + ) to inputs in $\mathcal{F}$. $\diamond$

It has been known since the 1980 s that $\mathbf{F E A S} \mathbf{S}_{\mathbb{R}} \in \mathbf{P S P A C E}$ Can88, and an NP-hardness lower bound was certainly known earlier. However, no sharper bounds in terms of sparsity were known earlier in the Turing model until our first main theorem.

ThEOREM 1.3. Let $Z_{+}(f)$ denote the zero set of $f$ in $\mathbb{R}_{+}^{n}$. Then:

$0 . \mathbf{F E A S}_{+}\left(\bigcup_{n \in \mathbb{N}} \mathcal{F}_{n, n+1}^{*}\right)$ and $\mathbf{F E A S}_{\mathbb{R}}\left(\bigcup_{n \in \mathbb{N}} \mathcal{F}_{n, n+1}^{*}\right)$ are in $\mathbf{N C}^{1}$. In particular, when $f \in \mathcal{F}_{n, n+1}^{*}, Z_{+}(f)$ is either empty or diffeotopi 1 to $\mathbb{R}_{+}^{n-1}$, with each case actually occuring.

1. For any fixed $n, \mathbf{F E A} \mathbf{S}_{+}\left(\mathcal{F}_{n, n+2}^{*}\right)$ and $\mathbf{F E A} \mathbf{S}_{\mathbb{R}}\left(\mathcal{F}_{n, n+2}^{*}\right)$ are in $\mathbf{P}$.

2. For any fixed $\varepsilon>0$, both $\mathbf{F E A S} \mathbf{S}_{+}\left(\bigcup_{n \in \mathbb{N}} \mathcal{F}_{n, n+n^{\varepsilon}}^{*}\right)$ and $\mathbf{F E A S}_{\mathbb{R}}\left(\bigcup_{n \in \mathbb{N}} \mathcal{F}_{n, n+n^{\varepsilon}}^{*}\right)$ are $\mathbf{N P}$-hard.

Slightly sharper algorithmic complexity bounds hold when we instead work in the BSS model over $\mathbb{R}$ (thus counting arithmetic operations instead of bit operations), and this is detailed in [PRT09.

Example 1.4. A very special case of Assertion (1) of Theorem 1.3 implies that one can decide - for any nonzero $c_{1}, \ldots, c_{5} \in \mathbb{Z}$ and $D \in \mathbb{N}$ - whether

$$
c_{1}+c_{2} x_{1}^{999}+c_{3} x_{1}^{73} x_{3}^{19}+c_{4} x_{2}^{27 D}+c_{5} x_{1}^{74} x_{2}^{D} x_{3}
$$

has a root in $\mathbb{R}^{3}$, using a number of bit operations polynomial in

$$
\log (D)+\log \left[\left(\left|c_{1}\right|+1\right) \cdots\left(\left|c_{5}\right|+1\right)\right]
$$

The best previous results (e.g., via the critical points method, infinitesimals, and rational univariate reduction, as detailed in [BPR06]) would yield a bound polynomial in $D+\log \left[\left(\left|c_{1}\right|+1\right) \cdots\left(\left|c_{5}\right|+1\right)\right]$ instead. $\diamond$

We thus see that for sparse polynomials, large degree can be far less of a complexity bottleneck over $\mathbb{R}$ than over $\mathbb{C}$. Theorem 1.3 is proved in Section 3.2 below. The underlying techniques include $\mathcal{A}$-discriminants (a.k.a. sparse discriminants) (cf. Section 2.3), Viro's Theorem from toric geometry (see the Appendix, or GKZ94, Thm. 5.6]), and effective estimates on linear forms in logarithms Bak77, Nes03.

In particular, for any collection $\mathcal{F}_{\mathcal{A}}$ of $n$-variate $m$-nomials with support $\mathcal{A}$, there is a polynomial $\Delta_{\mathcal{A}}$ in the coefficients $\left(c_{i}\right)$ called the $\mathcal{A}$-discriminant. Its real zero set partitions $\mathcal{F}_{\mathcal{A}}$ into chambers (connected components of the complement) on which the zero set of an $f \in \mathcal{F}_{\mathcal{A}}$ has constant topological type. A toric deformation argument employing Viro's Theorem enables us to decide whether a given chamber consists of $f$ having empty or non-empty $Z_{+}(f)$. For any $\mathcal{A} \subset \mathbb{Z}^{n}$ of cardinality $n+2$ (in sufficiently general position), there is then a compact formula for the $\mathcal{A}$-discriminant that enables us to pick out which chamber contains a given $f$ : one simply computes the sign of a linear combination of

\footnotetext{
${ }^{1}$ See Definition 2.10 of Section 2.3 below.
}

logarithms. Our resulting algorithms are thus quite implementable, requiring only fast approximation of logarithms and some basic triangulation combinatorics for $\operatorname{Supp}(f)$.

Example 1.5. Consider $\mathcal{A}:=\{(0,0,0),(999,0,0),(73,0,19)$, $(0,2009,0),(74,293,1)\}$, which gives us the family of trivariate pentanomials

$\mathcal{F}_{\mathcal{A}}:=\left\{c_{1}+c_{2} x_{1}^{999}+c_{3} x_{1}^{73} x_{3}^{19}+c_{4} x_{2}^{2009}+c_{5} x_{1}^{74} x_{2}^{293} x_{3} \mid c_{i} \in \mathbb{R}^{*}\right\}$. Suppose further that $f \in \mathcal{F}_{\mathcal{A}}$ is an element satisfying $c_{1}, c_{2}, c_{3}$, $c_{4}>0$ and $c_{5}<0$. It then turns out via Lemma 2.13 (cf. Section 2.4 below) that $Z_{+}(f)$ has a degeneracy iff the $\mathcal{A}$ discriminant, $\Delta_{\mathcal{A}}(c):=$ $38132829^{38132829} c_{1}^{27886408} c_{2}^{2677997} c_{3}^{2006991} c_{4}^{5561433}$

$-27886408^{27886408} 2677997^{2677997} 2006991^{2006991} 5561433^{5561433} c_{5}^{38132829}$

vanishes. In fact, via the techniques underlying Theorem 1.3. $Z_{+}(f)$ is either empty, a point, or isotopic to a 2-sphere, according as $\Delta_{\mathcal{A}}(c)$ is positive, zero, or negative. Note in particular that determining the sign of $\Delta_{\mathcal{A}}(c)$ is equivalent to determining the sign of

$38132829 \log (38132829)+27886408 \log \left(c_{1}\right)+2677997 \log \left(c_{2}\right)+2006991 \log \left(c_{3}\right)+5561433 \log \left(c_{4}\right)$ $-27886408 \log (27886408)-2677997 \log (2677997)-2006991 \log (2006991)-5561433 \log (5561433)-38132829 \log \left(c_{5}\right) . \diamond$

While we review $\mathcal{A}$-discriminants in Section 2.3 below, it is important to observe now how the computational complexity of $\mathcal{A}$-discriminants closely parallels that of $\mathbf{F E A S} \mathbf{S}_{\mathbb{R}}$ : compare Theorem 1.3 above with Theorem 1.7 below.

Definition 1.6. Let $\mathbf{A D I S C}_{=}$(resp. $\mathbf{A D I S C}_{>}$) denote the problem of deciding whether $\Delta_{\mathcal{A}}(f)$ vanishes (resp. determining the sign of $\Delta_{\mathcal{A}}(f)$ ) for an input polynomial $f$ with integer coefficients, where $\mathcal{A}=\operatorname{Supp}(f)$. Finally, let $\mathbf{A D I S C}_{=}(\mathcal{F})$ (resp. $\mathbf{A D I S C}_{>}(\mathcal{F})$ ) be the natural restriction of $\mathbf{A D I S C}=($ resp. $\mathbf{A D I S C})$ to inputs in some family $\mathcal{F}$.

THEOREM 1.7.

1. $\mathbf{A D I S C}_{=}\left(\bigcup_{n \in \mathbb{N}} \mathcal{F}_{n, n+2}^{*}\right) \in \mathbf{P}$ and, for any fixed $n$,
$\mathbf{A D I S C}_{>}\left(\mathcal{F}_{n, n+2}^{*}\right) \in \mathbf{P}$.

2. For any fixed $\varepsilon>0$, both $\mathbf{A D I S C}=\left(\bigcup_{n \in \mathbb{N}} \mathcal{F}_{n, n+n^{\varepsilon}}^{*}\right)$ and $\mathbf{A D I S C}_{>}\left(\bigcup_{n \in \mathbb{N}} \mathcal{F}_{n, n+n^{\varepsilon}}^{*}\right)$ are $\mathbf{N P}$-hard.

Theorem 1.7 is proved in Section 3.1 after the development of some necessary theory in Section 2 below.

\subsection{Related Work}

Earlier work on algorithmic fewnomial theory has mainly gone in directions other than polynomial-time algorithms. For example, Gabrielov and Vorobjov have given singly exponential time algorithms for weak stratifications of semi-Pfaffian sets GV04 — data from which one can compute homology groups of real zero sets of a class of functions more general than sparse polynomials. Our approach thus highlights a subproblem where faster and simpler algorithms are possible.

Focussing on feasibility, other than the elementary results $\mathbf{F E A S}_{\mathbb{R}}\left(\mathcal{F}_{1,1}\right) \in \mathbf{N} \mathbf{C}^{0}$ and $\mathbf{F E A} \mathbf{S}_{\mathbb{R}}\left(\mathcal{F}_{1,2}\right) \in \mathbf{N C}^{0}$, there appear to have been no earlier complexity upper bounds of the form $\mathbf{F E A} \mathbf{S}_{\mathbb{R}}\left(\mathcal{F}_{1, m}\right) \in \mathbf{P}$, or even $\mathbf{F E A} \mathbf{S}_{\mathbb{R}}\left(\mathcal{F}_{1, m}\right) \in \mathbf{N P}$, for $m \geq 3$. (With the exception of [RY05, algorithmic work on univariate real polynomials has focussed on algorithms that are quasi-linear in the degree. See, e.g., [LM01.) 
Echoing the parallels between $\mathbf{F E A} \mathbf{S}_{\mathbb{R}}$ and $\mathbf{A D I S C}>$ provided by Theorems 1.3 and 1.7 both $\mathbf{F E A S}_{\mathbb{R}}\left(\mathcal{F}_{1,4}\right) \stackrel{?}{\in} \mathbf{P}$ and $\mathbf{A D I S C}_{>}\left(\mathcal{F}_{1,4}\right) \stackrel{?}{\in} \mathbf{P}$ are open problems.

As for earlier complexity lower bounds for $\mathbf{F E A} \mathbf{S}_{\mathbb{R}}$ in terms of sparsity, we are unaware of any. Indeed, it is not even known whether $\mathbf{F E A} \mathbf{S}_{\mathbb{R}}\left(\mathbb{Z}\left[x_{1}, \ldots, x_{n}\right]\right)$ is $\mathbf{N P}$-hard for some fixed $n$. Also, complexity lower bounds for the vanishing of discriminants of $n$-variate $(n+k(n))$-nomials (with $k$ a slowly growing function of $n$ ) appear to be new. However, recent work shows that the geometry of discriminants chambers can be quite intricate already for $f \in \mathcal{F}_{3,3+3}^{*}$ [DRRS07. Also, it was known even earlier that deciding the vanishing of sparse discriminants of univariate $m$-nomials (with $m$ unbounded) is already NP-hard with respect to randomized reductions [KS99. Considering Theorems 1.3 and 1.7 one may thus be inclined to conjecture that $\mathbf{F E A S} \mathbf{S}_{\mathbb{R}}\left(\mathbb{Z}\left[x_{1}\right]\right)$ is NP-hard. Curiously, over a different family of complete fields (the $\boldsymbol{p}$-adic rationals), one can already prove that detecting roots for univariate $m$-nomials (with $m$ unbounded) is NP-hard with respect to randomized reductions [RR07.

\section{BACKGROUND AND ANCILLARY RESULTS}

After recalling a basic complexity construction, we will present some tools for dealing with $n$-variate $(n+1)$-nomials, and then move on to $n$-variate $(n+k)$-nomials with $k \geq 2$. All proofs for the results of this section are in the Appendix.

\subsection{A Key Reduction}

To measure the complexity of our algorithms, let us fix the following definitions for input size.

Definition 2.1. For any $a \in \mathbb{Z}$, we define its size, $\operatorname{size}(a)$, to be $1+\log (1+|a|)$. More generally, we define the size of a matrix $U=\left[u_{i, j}\right] \in \mathbb{Z}^{m \times n}$ to be $\sum_{i, j} \operatorname{size}\left(u_{i, j}\right)$. Also, for any $f(x)=\sum_{i=1}^{m} c_{i} x^{a_{i}} \in \mathbb{Z}\left[x_{1}, \ldots, x_{n}\right]$, we define size $(f)$ to be $\sum_{i=1}^{m}\left[\operatorname{size}\left(c_{i}\right)+\operatorname{size}\left(a_{i}\right)\right]$. Finally, for $F=\left(f_{1}, \ldots, f_{k}\right) \in$ $\left(\mathbb{Z}\left[x_{1}, \ldots, x_{n}\right]\right)^{k}$, we define $\operatorname{size}(F)=\sum_{i=1}^{k} \operatorname{size}\left(f_{i}\right)$.

A key construction we will use later in our NP-hardness proofs is a refinement of an old trick for embedding Boolean satisfiability into real/complex satisfiability. We refer to the well-known 3CNFSAT problem, reviewed in the Appendix.

Proposition 2.2. Given any 3CNFSAT instance $B(X)$ with $n$ variables and $N$ clauses, let $W_{B}$ denote $\left(\left(\{1\} \times \mathbb{P}_{\mathbb{C}}^{1}\right) \cup\left(\mathbb{P}_{\mathbb{C}}^{1} \times\{1\}\right)\right)^{4 N-n}$. Then there is an $(8 N-n) \times(8 N-n)$ polynomial system $F_{B}$ with the following properties:

1. $B(X)$ is satisfiable iff $F_{B}$ has a root in $\{1,2\}^{n} \times W_{B}$.

2. $F_{B}$ has no more than $33 N-4 n$ monomial terms, $\operatorname{size}\left(F_{B}\right)=O(N)$, and every root of $F_{B}$ in $\left(\mathbb{P}_{\mathbb{C}}^{1}\right)^{8 N-n}$ lies in $\{1,2\}^{n} \times W_{B}$ and is degenerate.

Also, if we define $t_{M}\left(z_{1}, \ldots, z_{M}\right)$ to be $1+z_{1}^{M+1}+\cdots+z_{M}^{M+1}-(M+1) z_{1} \cdots z_{M}$, then

3. $t_{M}$ is nonnegative on $\mathbb{R}_{+}^{M}$, with a unique positive root at $(1, \ldots, 1)$ that happens to be the only degenerate root of $t_{M}$ in $\mathbb{C}^{M}$.

$$
\begin{aligned}
& \text { 4. If } \varepsilon>0, f \in \mathcal{F}_{n, n+k}^{*} \text {, and } M:=\left[k^{1 / \varepsilon}\right] \text {, then } f(x)+ \\
& t_{M}(z) \in \mathcal{F}_{\eta, \eta+\eta^{\delta}}^{*} \text { for } \eta=n+M \text { and some positive } \delta \leq \varepsilon \text {. } \\
& \text { In particular, } \operatorname{size}\left(f(x)+t_{M}(z)\right)=O\left(\operatorname{size}(f)^{1 / \varepsilon}\right) \text {. }
\end{aligned}
$$

The seemingly mysterious polynomial $t_{M}$ defined above will be useful later when we will need to decrease the difference between the number of terms and variables in certain polynomials.

\subsection{Efficient Linear Algebra on Exponents}

A simple and useful change of variables is to use monomials in new variables.

DeFinition 2.3. For any ring $R$, let $R^{m \times n}$ denote the set of $m \times n$ matrices with entries in $R$. For any $M=\left[m_{i j}\right] \in$ $\mathbb{R}^{n \times n}$ and $y=\left(y_{1}, \ldots, y_{n}\right)$, we define the formal expression $y^{M}:=\left(y_{1}^{m_{1,1}} \cdots y_{n}^{m_{n, 1}}, \ldots, y_{1}^{m_{1, n}} \cdots y_{n}^{m_{n, n}}\right)$. We call the substitution $x:=y^{M}$ a monomial change of variables. Also, for any $z:=\left(z_{1}, \ldots, z_{n}\right)$, we let $x z:=\left(x_{1} z_{1}, \ldots, x_{n} z_{n}\right)$. Finally, let $\mathbb{G L}_{n}(\mathbb{Z})$ denote the group of all matrices in $\mathbb{Z}^{n \times n}$ with determinant \pm 1 (the set of unimodular matrices). $\diamond$

Proposition 2.4. (See, e.g., LRW03, Prop. 2].) For any $U, V \in \mathbb{R}^{n \times n}$, we have the formal identity $(x y)^{U V}=$ $\left(x^{U}\right)^{V}\left(y^{U}\right)^{V}$. Also, if $\operatorname{det} U \neq 0$, then the function $e_{U}(x):=x^{U}$ is an analytic automorphism of $\mathbb{R}_{+}^{n}$, and preserves smooth points and singular points of positive zero sets of analytic functions. Moreover, if $\operatorname{det} U>0$, then $e_{U}$ in fact induces a diffeotopy on any positive zero set of an analytic function. Finally, $U \in \mathbb{G L}_{n}(\mathbb{R})$ implies that $e_{U}^{-1}\left(\mathbb{R}_{+}^{n}\right)=\mathbb{R}_{+}^{n}$ and that $e_{U}$ maps distinct open orthants of $\mathbb{R}^{n}$ to distinct open orthants of $\mathbb{R}^{n}$.

Proposition 2.4 with minor variations, has been observed in many earlier works (see, e.g., LRW03). Perhaps the only new ingredient is the observation on diffeotopy, which follows easily from the fact that $\mathbb{G L}_{n}^{+}(\mathbb{R})$ (the set of all $n \times n$ real matrices with positive determinant) is a connected Lie group.

Recall that the affine span of a point set $\mathcal{A} \subset \mathbb{R}^{n}, \operatorname{Aff} \mathcal{A}$, is the set of real linear combinations $\sum_{a \in \mathcal{A}} c_{a} a$ satisfying $\sum_{a \in \mathcal{A}} c_{a}=0$.

Lemma 2.5. Given any $f \in \mathcal{F}_{n, m}$ with $d=$ $\operatorname{dim} \operatorname{Aff}(\operatorname{Supp}(f))<n<m$, we can find (using a number of bit operations polynomial in $\operatorname{size}(\operatorname{Supp}(f)))$ a $U \in \mathbb{G L}_{n}(\mathbb{Z})$ such that $g(y):=f\left(y^{U}\right) \in \mathcal{F}_{d, m}^{*}$ and $g$ vanishes in $\mathbb{R}_{+}^{d}$ (resp. $\left.\left(\mathbb{R}^{*}\right)^{d}\right)$ iff $f$ vanishes in $\mathbb{R}_{+}^{n}\left(\right.$ resp. $\left.\left(\mathbb{R}^{*}\right)^{n}\right)$. In particular, there is an absolute constant $c$ such that $\operatorname{size}(U)=$ $O\left(\operatorname{size}(\operatorname{Supp}(f))^{c}\right)$.

To study $Z_{+}(f)$ when $f \in \mathcal{F}_{n, n+1}^{*}$ it will help to have a much simpler canonical form. In what follows, we use \# for set cardinality and $e_{i}$ for the $i$ th standard basis vector of $\mathbb{R}^{n}$.

LemMa 2.6. For any $f \in \mathcal{F}_{n, n+1}^{*}$ we can compute $\ell \in$ $\{0, \ldots, n\}$ within $\mathbf{N C}^{1}$ and $\gamma \in \mathbb{R}+$ such that $\bar{f}(x):=\gamma+$ $x_{1}+\cdots+x_{\ell}-x_{\ell+1}-\cdots-x_{n}$ satisfies: (1) either $f$ or $-f$ has exactly $\ell+1$ positive coefficients, and (2) $Z_{+}(\bar{f})$ and $Z_{+}(f)$ are diffeotopic.

Corollary 2.7. Suppose $f \in \mathcal{F}_{n, n+1}^{*}$ and $\operatorname{Supp}(f)=$ $\left\{a_{1}, \ldots, a_{n+1}\right\} \subset \mathbb{R}^{n}$. Then 
1. $f$ has a root in $\mathbb{R}_{+}^{n} \Longleftrightarrow$ not all the coefficients of $f$ have the same sign. In particular, $Z_{+}(f)$ is diffeotopic to either $\mathbb{R}_{+}^{n-1}$ or $\emptyset$.

2. If all the coefficients of $f$ have the same sign, then $f$ has a root in $\left(\mathbb{R}^{*}\right)^{n} \Longleftrightarrow$ there are indices $i \in[n]$ and $j, j^{\prime} \in[n+1]$ with $a_{i, j}-a_{i, j^{\prime}}$ odd.

\subsection{Combinatorics and Topology of Certain $\mathcal{A}$ - Discriminants}

The connection between topology of discriminant complements and computational complexity dates back to the late 1970s, having been observed relative to (a) the membership problem for semi-algebraic sets [DL79] and (b) the approximation of roots of univariate polynomials Sma87. Our goal here is a precise connection between $\mathbf{F E A} \mathbf{S}_{\mathbb{R}}$ and $\mathcal{A}$ discriminant complements. (See also [DRRS07] for further results in this direction.)

Definition 2.8. GKZ94, Ch. 1 \& 9-11] Given any $\mathcal{A}=$ $\left\{a_{1}, \ldots, a_{m}\right\} \subset \mathbb{Z}^{n}$ of cardinality $m$ and $c_{1}, \ldots, c_{m} \in \mathbb{C}^{*}$, we define $\nabla_{\mathcal{A}} \subset \mathbb{P}_{\mathbb{C}}^{m-1} \quad-$ the $\mathcal{A}$-discriminant variety - to be the closure of the set of all $\left[c_{1}: \cdots: c_{m}\right] \in \mathbb{P}_{\mathbb{C}}^{m-1}$ such that $f(x)=\sum_{i=1}^{m} c_{i} x^{a_{i}}$ has a degenerate root in $\mathbb{C}^{n}$. We then define $\Delta_{\mathcal{A}} \in \mathbb{Z}\left[c_{1}, \ldots, c_{m}\right] \backslash\{0\}$ - the $\mathcal{A}$-discriminant - to be the unique (up to sign) irreducible defining polynomial of $\nabla_{\mathcal{A}}$. Also, when $\nabla_{\mathcal{A}}$ has complex codimension at least 2 , we set $\Delta_{\mathcal{A}}$ to the constant 1. For convenience, we will sometimes write $\Delta_{\mathcal{A}}(f)$ in place of $\Delta_{\mathcal{A}}\left(c_{1}, \ldots, c_{m}\right)$.

To prove our results, it will actually suffice to deal with a small subclass of $\mathcal{A}$-discriminants.

Definition 2.9. We call $\mathcal{A} \subset \mathbb{R}^{n}$ a (non-degenerate) circuit ${ }^{2}$ iff $\mathcal{A}$ is affinely dependent, but every proper subset of $\mathcal{A}$ is affinely independent. Also, we say that $\mathcal{A}$ is a degenerate circuit iff $\mathcal{A}$ contains a point a and a proper subset $\mathcal{B}$ such that $a \in B, \mathcal{A} \backslash a$ is affinely independent, and $\mathcal{B}$ is a non-degenerate circuit. $\diamond$

For instance, both $\therefore$ and $\because$ are circuits, but.. is a degenerate circuit. In general, for any degenerate circuit $\mathcal{A}$, the subset $\mathcal{B}$ named above is always unique.

The relevance of $\mathcal{A}$-discriminants to $m$-nomial zero sets can be summarized as follows.

Definition 2.10. Following the notation of Definition 2.8 we call any connected component of $\mathbb{P}_{\mathbb{R}}^{m-1} \backslash\left(\nabla_{\mathcal{A}} \cup\left\{\left[x_{1}: \cdots\right.\right.\right.$ : $\left.\left.\left.x_{m}\right] \mid x_{1} \cdots x_{m}=0\right\}\right)$ a (real) $\mathcal{A}$-discriminant chamber. Also, given any subsets $X, Y \subseteq \mathbb{R}_{+}^{n}$, we say that they are isotopic (resp. diffeotopic) iff there is a continuous (resp. differentiable) function $H:[0,1] \times X \longrightarrow \mathbb{R}_{+}^{n}$ such that $H(t, \cdot)$ is a homeomorphism (resp. diffeomorphism) for all $t \in[0,1]$, $H(0, \cdot)$ is the identity on $X$, and $H(1, X)=Y$. Finally, for any $\mathcal{A} \subset \mathbb{R}^{n}$ of cardinality $m$, let $\mathcal{F}_{\mathcal{A}}$ denote the set of all $n$-variate $m$-nomials with support $\mathcal{A}$. $\diamond$

REMARK 2.11. Note that when $\mathcal{A}$ has cardinality $m$, we may naturally identify elements of $\mathbb{P}_{\mathbb{C}}^{m-1}$ (resp. $\mathbb{P}_{\mathbb{R}}^{m-1}$ ) with equivalence classes determined by nonzero complex (resp. real) multiples of elements of $\mathcal{F}_{\mathcal{A}}$. $\diamond$

\footnotetext{
${ }^{2}$ This terminology comes from matroid theory and has nothing to do with circuits from complexity theory.
}

The topology of toric real zero sets is known to be constant on discriminant chambers (see, e.g., GKZ94, Ch. 11, Sec. 5A, Prop. 5.2, pg. 382]). However, we will need a refinement of this fact to positive zero sets, so we derive this directly for $\mathcal{A}$ in sufficiently general position - non-degenerate circuits in particular.

Lemma 2.12. Following the notation above, suppose $\mathcal{A} \subset$ $\mathbb{R}^{n}$ is such that the minimum of any linear form on $\mathcal{A}$ is minimized at no more than $n+1$ points. Also let $\mathcal{C}$ be any $\mathcal{A}$ discriminant chamber. Then $f, g \in \mathcal{C} \Longrightarrow Z_{+}(f)$ and $Z_{+}(g)$ are diffeotopic.

There is then a very compact description for $\nabla_{\mathcal{A}}$ when $\mathcal{A}$ is a circuit.

Lemma 2.13. Suppose $\mathcal{A}=\left\{a_{1}, \ldots, a_{n+2}\right\} \subset \mathbb{Z}^{n}$ is a nondegenerate circuit, $f$ is a polynomial with support $\mathcal{A}, \hat{\mathcal{A}}$ is the $(n+1) \times(n+2)$ matrix whose $j^{\text {th }}$ column is $\{1\} \times a_{j}, \hat{\mathcal{A}}_{j}$ is the submatrix of $\hat{\mathcal{A}}$ obtained by deleting the $j^{\text {th }}$ column, and $b_{j}:=(-1)^{j} \operatorname{det} \hat{\mathcal{A}}_{j} / \beta$ where $\beta=\operatorname{gcd}\left(\operatorname{det} \hat{\mathcal{A}}_{1}, \ldots, \operatorname{det} \hat{\mathcal{A}}_{n+2}\right)$. Then:

1. $\Delta\left(c_{1}, \ldots, c_{n+2}\right)$ is, up to a multiple by a nonzero monomial term, $\prod_{i=1}^{n+2}\left(\frac{c_{i}}{b_{i}}\right)^{b_{i}}-1$. Also, $\left(b_{1}, \ldots, b_{n+2}\right)$ can be computed in $\mathbf{P}$.

2. $\prod_{i=1}^{n+2}\left(\operatorname{sign}\left(b_{i} c_{i}\right) c_{i} / b_{i}\right)^{\operatorname{sign}\left(b_{i} c_{i}\right) b_{i}}=1$ for some $\left[c_{1}: \cdots\right.$ : $\left.c_{n+2}\right] \in \mathbb{P}_{\mathbb{R}}^{n+1}$ with $\operatorname{sign}\left(c_{1} b_{1}\right)=\cdots=\operatorname{sign}\left(c_{n+2} b_{n+2}\right) \Longleftrightarrow$ $Z_{+}\left(\sum_{i=1}^{n+2} c_{i} x^{a_{i}}\right)$ contains a degenerate point $\zeta$. In particular, $Z_{+}(f)$ has at most one degenerate point.

3. $\mathcal{A}$ has exactly two triangulations: one with simplices $\left\{\operatorname{Conv}\left(\mathcal{A} \backslash\left\{b_{i}\right\}\right) \mid \operatorname{sign}\left(b_{i}\right)>0\right\}$, and the other with simplices $\left\{\operatorname{Conv}\left(\mathcal{A} \backslash\left\{b_{i}\right\}\right) \mid \operatorname{sign}\left(b_{i}\right)<0\right\}$. Moreover, the preceding description also holds when $\mathcal{A}$ is a degenerate circuit.

\subsection{Complexity of Circuit Discriminants and Linear Forms in Logarithms}

Theorem [1.7 is a central tool behind the upper bounds and lower bounds of Theorem 1.3 , and is precisely where diophantine approximation enters our scenery. To wit, the proof of Assertion (1) of Theorem 1.7 makes use of the following powerful result.

Nesterenko-Matveev Theorem. [Nes03, Thm. 2.1, Pg. 55] For any integers $c_{1}, \alpha_{1}, \ldots, c_{N}, \alpha_{N}$ with $\alpha_{i} \geq 2$ for all $i$, define $\Lambda(c, \alpha):=c_{1} \log \left(\alpha_{1}\right)+\cdots+c_{N} \log \left(\alpha_{N}\right)$. Then $\Lambda(c, \alpha) \neq 0 \Longrightarrow \log \left|\frac{1}{\Lambda(c, \alpha)}\right|$ is bounded above by

$$
2.9(N+2)^{9 / 2}(2 e)^{2 N+6}\left(2+\log \max _{j}\left|c_{j}\right|\right) \prod_{j=1}^{N} \log \left|\alpha_{j}\right| .
$$

Assertion (1) of Theorem 1.7 will follow easily from the two algorithms we state below, once we prove their correctness and verify their efficiency. However, we will first need to recall the concept of a gcd-free basis. In essence, a gcdfree basis is nearly as powerful as factorization into primes, but is far easier to compute.

Definition 2.14. BS96, Sec. 8.4] For any subset $\left\{\alpha_{1}, \ldots, \alpha_{N}\right\}$ $\subset \mathbb{N}$, a gcd-free basis is a pair of sets $\left(\left\{\gamma_{i}\right\}_{i=1}^{\eta},\left\{e_{i j}\right\}_{(i, j) \in[N] \times[\eta]}\right)$ such that (1) $\operatorname{gcd}\left(\gamma_{i}, \gamma_{j}\right)=1$ for all $i \neq j$, and (2) $\alpha_{i}=$ $\prod_{j=1}^{\eta} \gamma^{e_{i j}}$ for all $i$. $\diamond$ 
Algorithm 2.15 .

Input: Integers $\alpha_{1}, \beta_{1}, u_{1}, v_{1}, \ldots, \alpha_{N}, \beta_{N}, u_{N}, v_{N}$. Output: A true declaration as to whether $\alpha_{1}^{u_{1}} \cdots \alpha_{N}^{u_{N}}=$ $\beta_{1}^{v_{1}} \cdots \beta_{N}^{v_{N}}$.

Description:

0. If $\prod_{i=1}^{N}\left(\operatorname{sign} \alpha_{i}\right)^{u_{i} \bmod 2} \neq \prod_{i=1}^{N}\left(\operatorname{sign} \beta_{i}\right)^{v_{i} \bmod 2}$ then output "They are not equal." and STOP.

1. Replace the $\alpha_{i}$ and $\beta_{i}$ by their absolute values and then construct, via Theorem 4.9 of the Appendix, a gcdfree basis $\left(\left\{\gamma_{i}\right\}_{i=1}^{\eta},\left\{e_{i j}\right\}_{(i, j) \in[2 N] \times[\eta]}\right)$ for $\alpha_{1}, \ldots, \alpha_{N}$, $\beta_{1}, \ldots, \beta_{N}$

2. If $\sum_{i=1}^{N} e_{i j} u_{i}=\sum_{i=N+1}^{2 N} e_{i j} v_{i}$ for all $j \in[\eta]$ then output "They are equal." and STOP.

3. Output "They are not equal."

AlgORITHM 2.16 .

Input: Positive integers $\alpha_{1}, u_{1}, \ldots, \alpha_{M}, u_{M}$ and $\beta_{1}, v_{1}$, $\ldots, \beta_{N}, v_{N}$ with $\alpha_{i}, \beta_{i} \geq 2$ for all $i$.

Output: The sign of $\alpha_{1}^{u_{1}} \cdots \alpha_{M}^{u_{M}}-\beta_{1}^{v_{1}} \cdots \beta_{N}^{v_{N}}$.

Description:

0. Check via Algorithm 2.15 whether $\alpha_{1}^{u_{1}} \cdots \alpha_{M}^{u_{M}}=\beta_{1}^{v_{1}} \cdots \beta_{N}^{v_{N}}$. If so, output "They are equal." and STOP.

1. Let $U:=\max \left\{u_{1}, \ldots, u_{M}, v_{1}, \ldots, v_{N}\right\}$, and $E:=\frac{2.9}{\log 2}(2 e)^{2 M+2 N+6}(1+\log U)$

$\times\left(\prod_{i=1}^{M} \log \left|\alpha_{i}\right|\right)\left(\prod_{i=1}^{N} \log \left|\beta_{i}\right|\right)$.

2. For all $i \in[M]$ (resp. $i \in[N])$, let $A_{i}$ (resp. $\left.B_{i}\right)$ be a rational number agreeing with $\log \alpha_{i}\left(\right.$ resp. $\left.\log \beta_{i}\right)$ in its first $2+E+\log _{2} M$ (resp. $2+E+\log _{2} N$ ) leading bits $3^{3}$

3. Output the sign of $\left(\sum_{i=1}^{M} u_{i} A_{i}\right)-\left(\sum_{i=1}^{N} v_{i} B_{i}\right)$ and STOP.

Lemma 2.17. Algorithms 2.15 and 2.16 are both correct. Moreover, following the preceding notation, Algorithms 2.15 and 2.16 run within a number of bit operations asymptotically linear in, respectively,

$$
\sum_{i=1}^{N}\left(\log \left(u_{i}\right) \log \left(\alpha_{i}\right)+\log \left(v_{i}\right) \log \left(\beta_{i}\right)\right)^{2}
$$

and

$(M+N)(30)^{M+N} L(\log U)\left(\prod_{i=1}^{M} L\left(\log \left(\alpha_{i}\right)\right)\right)\left(\prod_{i=1}^{N} L\left(\log \left(\beta_{i}\right)\right)\right)$, where $L(x):=x \log ^{2}(x) \log \log (x)$.

\subsection{Positive Feasibility for Circuits}

For a real polynomial supported on a non-degenerate circuit, there are just two ways it can fail to have a positive root: a simple way and a subtle way. This is summarized below. Recall that the Newton polytope of $f$ is simply $\operatorname{Newt}(f):=\operatorname{Conv}(\operatorname{Supp}(f))$, where $\operatorname{Conv}(S)$ denotes the convex hull (smallest convex set) containing $S$.

ThEOREM 2.18. Suppose $f(x)=\sum_{i=1}^{n+2} c_{i} x^{a_{i}} \in \mathcal{F}_{n, n+2}^{*}$, $\operatorname{Supp}(f)$ is a non-degenerate circuit, and $b$ is the vector from Lemma 2.13. Then $Z_{+}(f)$ is empty iff one of the following conditions holds:

${ }^{3}$ For definiteness, let us use Arithmetic-Geometric Mean Iteration as in Ber03 to find these approximations.
1. All the $c_{i}$ have the same sign.

2. Newt $(f)$ is an $n$-simplex and, assuming $a_{j^{\prime}}$ is the unique element of $\mathcal{A}$ lying in the interior of $\operatorname{Newt}(f)$, we have $-\operatorname{sign}\left(c_{j^{\prime}}\right)=\operatorname{sign}\left(c_{i}\right)$ for all $i \neq j^{\prime}$ and $\prod_{i=1}^{n+2}\left(\operatorname{sign}\left(b_{j^{\prime}} c_{j^{\prime}}\right) \frac{c_{i}}{b_{i}}\right)^{\operatorname{sign}\left(b_{j^{\prime}} c_{j^{\prime}}\right) b_{i}}>1$.

Positive feasibility for polynomials supported on degenerate circuits can then essentially be reduced to the nondegenerate circuit case in some lower dimension. An additional twist arises from the fact that the zero sets of polynomials supported on degenerate circuits are, up to a monomial change of variables, the graphs of polynomials supported on non-degenerate circuits.

TheOREM 2.19. Suppose $f(x)=\sum_{i=1}^{n+2} c_{i} x^{a_{i}} \in \mathcal{F}_{n, n+2}^{*}$ has support $\mathcal{A} \subset \mathbb{R}^{n}$ that is a degenerate circuit with non-degenerate subcircuit $\mathcal{B}=\left\{a_{1}, \ldots, a_{j^{\prime}}\right\}$, and $b$ is the vector defined in Lemma 2.13 (ignoring the non-degeneracy assumption for $\mathcal{A})$. Then, when not all the coefficients of $f$ have the same sign, $Z_{+}(f)$ is empty iff both the following conditions hold:

a. $\operatorname{Conv}(\mathcal{B})$ is a $\left(j^{\prime}-2\right)$-simplex and, permuting indices so that $a_{j^{\prime}}$ is the unique element of $\mathcal{B}$ lying in the relative interior of $\operatorname{Conv}(\mathcal{B})$, we have $-\operatorname{sign}\left(c_{j^{\prime}}\right)=\operatorname{sign}\left(c_{i}\right)$ for all $i \neq j^{\prime}$.

b. $\prod_{i=1}^{j^{\prime}}\left(\operatorname{sign}\left(b_{j} c_{j}\right) \frac{c_{i}}{b_{i}}\right)^{\operatorname{sign}\left(b_{j} c_{j}\right) b_{i}} \geq 1$.

\section{THE PROOFS OF OUR MAIN RESULTS: THEOREMS 1.7 AND 1.3}

We go in increasing order of proof length.

\subsection{Proving Theorem 1.7}

Assertion (1): First note that any input $f$ must have support $\mathcal{A}=\left\{a_{1}, \ldots, a_{n+2}\right\}$ equal to either a degenerate circuit or a non-degenerate circuit. Recalling Assertion (1) of Lemma 2.13 observe then that the vector $b:=\left(b_{1}, \ldots, b_{n+2}\right)$ has a zero coordinate iff $\mathcal{A}$ is a degenerate circuit, and $b$ can be computed in time polynomial in $\operatorname{size}(\mathcal{A})$. If $\mathcal{A}$ is a degenerate circuit then (following easily from the definition) $\Delta_{\mathcal{A}}$ must be identically 1, thus leaving Assertion (1) of our present theorem trivially true. So let us assume henceforth that $\mathcal{A}$ is a non-degenerate circuit, and that $c_{j}$ is the coefficient of $x^{a_{j}}$ in $f$ for all $j$.

Via Assertion (1) of Lemma 2.13 once again, Assertion (1) of Theorem 1.7 follows routinely from the complexity bounds from Lemma 2.17. In particular, the latter lemma tells us that the bit complexity of $\mathbf{A D I S C}_{=}$, for input coefficients $\left(c_{1}, \ldots, c_{n+2}\right)$, is polynomial in $\sum_{i=1}^{n+2} \log \left(c_{i} b_{i}\right)$ (following the notation of Lemma 2.13); and the same is true for $\mathbf{A D I S C}_{>}$provided $n$ is fixed. The classical Hadamard inequality Mig92 then tells us that $\operatorname{size}\left(b_{i}\right)$ is $O\left(n \log \left(n \max _{j, k}\left\{a_{j k}\right\}\right)\right)$. So the complexity of $\mathbf{A D I S C}=$ is indeed polynomial in size $(f)$; and the same holds for ADISC $_{>}$when $n$ is fixed.

Assertion (2): We will construct an explicit reduction of 3CNFSAT to $\mathbf{A D I S C}_{=}\left(\bigcup_{n \in \mathbb{N}} \mathcal{F}_{n, n+n^{\varepsilon}}^{*}\right)$. In particular, to any 3CNFSAT instance $B(X)$ with $N$ clauses and $n$ variables, let us first consider $F_{B}=\left(f_{1}, \ldots, f_{8 N-n}\right)$ - the associated $(8 N-n) \times(8 N-n)$ polynomial system as detailed in Definition 4.1 of the Appendix and Proposition 2.2 of Section 2.1 
Let us then set $M:=\left\lceil(\max \{0,17 N-2 n\}+2)^{1 / \varepsilon}\right\rceil$ and define the single polynomial $f_{B}$ to be $f_{1}+\lambda_{1} f_{2}+\cdots+\lambda_{8 N-n-1} f_{8 N-n}+\lambda_{8 N-n} t_{M}\left(z_{1}, \ldots, z_{M}\right)$. Letting $\mathcal{A}$ be the support of $f_{B}$, it is then easily checked (from Definition 4.1 of the Appendix and Proposition 2.2) that $\mathcal{A}$ is affinely independent and $f_{B}$ is in $\mathcal{F}_{16 N-2 n+M, N^{\prime}}^{*}$ for some $N^{\prime} \leq 33 N-4 n+M+2$.

By the Cayley Trick GKZ94, Prop. 1.7, pp. 274] we then obtain that $\Delta_{\mathcal{A}}\left(f_{B}\right)=0$ iff

$(\star) F_{B}$ has a degenerate root in $\left(\mathbb{P}_{\mathbb{C}}^{1}\right)^{2 N-n}$ and $t_{M}$ has a degenerate root in $\left(\mathbb{C}^{*}\right)^{M}$

(Since Newt $\left(t_{M}\right)$ is a simplex, it is easily checked that $t_{M}$ has no complex degenerate roots at infinity.) By Proposition 2.2 the degenerate roots of $F_{B}$ are exactly $\{1,2\}^{n} \times W_{B}$, and $t_{M}$ has a unique degenerate root by construction. So $(\star)$ holds iff $B(X)$ has a satisfying assignment. We have thus reduced 3CNFSAT to detecting the vanishing of a particular $\mathcal{A}$-discriminant.

To conclude, observe that the number of terms of $f_{B}$ is only slightly larger than its number of variables, thanks to Proposition 2.2 In particular, $\operatorname{size}\left(f_{B}\right)=O\left(\operatorname{size}(B)^{1 / \varepsilon}\right)$ and $f_{B} \in \bigcup_{n \in \mathbb{N}} \mathcal{F}_{n, n+n^{\delta}}^{*}$ for some $\delta \in(0, \varepsilon]$. Clearly then, $\mathbf{A D I S C}_{=}\left(\bigcup_{n \in \mathbb{N}} \mathcal{F}_{n, n+n^{\varepsilon}}^{*}\right) \in \mathbf{P} \Longrightarrow \mathbf{P}=\mathbf{N P}$, thus proving our first desired NP-hardness lower bound.

The NP-hardness of ADISC $>\left(\bigcup_{n \in \mathbb{N}} \mathcal{F}_{n, n+n^{\delta}}^{*}\right)$ then follows immediately since $\mathbf{A D I S C}\left(\bigcup_{n \in \mathbb{N}} \mathcal{F}_{n, n+n^{\delta}}^{*}\right)$ is a refinement of $\mathbf{A D I S C}=\left(\bigcup_{n \in \mathbb{N}} \mathcal{F}_{n, n+n^{\delta}}^{*}\right)$.

\subsection{Proving Theorem 1.3}

Assertion (2): We will give an explicit reduction of 3CNFSAT to $\mathbf{F E A S} \mathbf{S}_{+}\left(\bigcup_{n \in \mathbb{N}} \mathcal{F}_{n, n+n^{\varepsilon}}^{*}\right)$. Attaining such a reduction will require little effort, thanks to our earlier reduction used to prove Assertion (2) of Theorem 1.7

In particular, for any 3CNFSAT instance $B$ with $N$ clauses and $n$ variables, let us recall the system $F_{B}=\left(f_{1}, \ldots, f_{8 N-n}\right)$ from Definition 4.1 of the Appendix and Proposition 2.2. Let us then define $M$ to be $\left\lceil(\max \{0,42 N-n\}+2)^{1 / \varepsilon}\right\rceil$ and define $g_{B}(x, z)$ to be $f_{1}^{2}(x)+\cdots+f_{4 N}^{2}(x)+t_{M}\left(z_{1}, \ldots, z_{M}\right)$. It is then easily checked that $f_{B} \in \mathcal{F}_{n+M, N^{\prime}}^{*}$ for some $N^{\prime} \leq 42 N+M+2$. Moreover, $B$ has a satisfying assignment iff $g_{B}$ has a positive root. (Indeed, any root of $g_{B}$ clearly lies in $\{1,2\}^{n} \times$ $\{1\}^{M}$.) We have thus reduced 3CNFSAT to a special case of $\mathbf{F E A S}$.

Now observe that the number of terms of $g_{B}$ is only slightly larger than its number of variables, thanks to Proposition 2.2 In particular, $\operatorname{size}\left(g_{B}\right)=O\left(\operatorname{size}(B)^{1 / \varepsilon}\right)$ and $g_{B}$ is in $\bigcup_{n \in \mathbb{N}} \mathcal{F}_{n, n+n^{\delta}}^{*}$ for some $\delta \in(0, \varepsilon]$. Clearly then, $\mathbf{F E A S}_{+}\left(\bigcup_{n \in \mathbb{N}} \mathcal{F}_{n, n+n^{\varepsilon}}^{*}\right) \in \mathbf{P} \Longrightarrow \mathbf{P}=\mathbf{N P}$, thus proving one of our desired NP-hardness lower bounds.

To conclude, we now need to prove the NP-hardness of $\mathbf{F E A S}_{\mathbb{R}}\left(\bigcup_{n \in \mathbb{N}} \mathcal{F}_{n, n+n^{\varepsilon}}^{*}\right)$. This we do by employing our preceding argument almost verbatim. The only difference is that we instead use the polynomial $h_{B}(x, z):=f_{1}^{2}(x)+\cdots+$ $f_{4 N}^{2}(x)+t_{M}\left(z_{1}^{2}, \ldots, z_{M}^{2}\right)$, and observe that $t_{M}\left(z_{1}^{2}, \ldots, z_{M}^{2}\right)$ is nonnegative on all of $\mathbb{R}^{n}$. So we are done.

Assertion (0): Our topological assertion follows immediately from Lemma 2.6 and Corollary 2.7

To obtain our algorithmic assertions, simply note that by
Assertion (1) of Corollary 2.7] detecting positive roots for $f$ reduces to checking whether all the coefficients have the same sign. This can clearly be done by $n$ sign evaluations and $n-1$ comparisons, doable in logarithmic parallel time. So the inclusion involving $\mathbf{F E A \mathbf { S } _ { + }}$ is proved.

Let us now show that we can detect roots in $\left(\mathbb{R}^{*}\right)^{n}$ within $\mathbf{N C}^{1}$ : Employing our algorithm from the last paragraph, we can clearly assume the signs of the coefficients of $f$ are all identical (for otherwise, we would have detected a root in $\mathbb{R}_{+}^{n}$ and finished). So then, by Assertion (2) of Corollary 2.7. we can simply do a parity check (trivially doable in $\mathbf{N C}^{1}$ ) of the entries of $\left[a_{2}-a_{1}, \ldots, a_{n+1}-a_{1}\right]$.

To conclude, we simply observe that our algorithm for detecting roots in $\left(\mathbb{R}^{*}\right)^{n}$ trivially extends to root detection in $\mathbb{R}^{n}$ : Any root of $f$ in $\mathbb{R}^{n}$ must lie in some coordinate subspace $L$ of minimal positive dimension. So, on $L$, the honest $n$-variate $(n+1)$-nomial $f$ will restrict to an $f^{\prime} \in$ $\mathcal{F}_{n^{\prime}, n^{\prime}+1}^{*}$ with $n^{\prime} \leq n$ and support a subset of the columns of a submatrix of $\mathcal{A}$. So then, we must check whether (a) all the coefficients of $f^{\prime}$ have the same sign or (if not), (b) a submatrix of $\left[a_{2}-a_{1}, \ldots, a_{n+1}-a_{1}\right]$ has an odd entry. In other words, $f$ has a root in $\mathbb{R}^{n} \Longleftrightarrow f$ has a root in $\left(\mathbb{R}^{*}\right)^{n} \cup\{\mathbf{O}\}$. Since checking whether $f$ vanishes at $\mathbf{O}$ is the same as checking whether $f$ is missing a constant term, checking for roots in $\mathbb{R}^{n}$ is thus also in $\mathbf{N C}^{1}$.

REMARK 3.1. Note that checking whether a given $f \in$ $\mathcal{F}_{n, n+1}$ lies in $\mathcal{F}_{n, n+1}^{*}$ can be done within $\mathbf{N C}^{2}$ : one simply finds $d=\operatorname{dim} \operatorname{Supp}(f)$ in $\mathbf{N C}^{2}$ by computing the rank of the matrix whose columns are $a_{2}-a_{1}, \ldots, a_{m}-a_{1}$ (via the parallel algorithm of Csanky [Csa76]), and then checks whether $d=n$. $\diamond$

Assertion (1): The algorithm we use to prove $\mathbf{F E A S}_{+}\left(\mathcal{F}_{n, n+2}^{*}\right)$ $\in \mathbf{P}$ for fixed $n$ is described just below. Note also that once we have $\mathbf{F E A S} \mathbf{S}_{+}\left(\mathcal{F}_{n, n+2}^{*}\right) \in \mathbf{P}$ for fixed $n$, it easily follows that $\mathbf{F E A} \mathbf{S}_{\mathbb{R}}\left(\mathcal{F}_{n, n+2}^{*}\right) \in \mathbf{P}$ : The polynomial obtained from an $f \in \mathcal{F}_{n, n+2}^{*}$ by setting any non-empty subset of its variables to 0 clearly lies in $\mathcal{F}_{n^{\prime}, n^{\prime}+2}^{*}$ for some $n^{\prime}<n$ (modulo a permutation of variables). Thus, since we can apply changes of variables like $x_{i} \mapsto-x_{i}$ in $\mathbf{P}$, and since there are exactly $3^{n}$ sequences of the form $\left(\varepsilon_{1}, \ldots, \varepsilon_{n}\right)$ with $\varepsilon_{i} \in\{0, \pm 1\}$ for all $i$, it thus clearly suffices to show that $\mathbf{F E A S} \mathbf{S}_{+}\left(\mathcal{F}_{n, n+2}^{*}\right) \in \mathbf{P}$ for fixed $n$.

We thus need only prove correctness, and a suitable complexity bound, for the following algorithm:

Algorithm 3.2.

Input: $A$ coefficient vector $c:=\left(c_{1}, \ldots, c_{n+2}\right)$ and a (possibly degenerate) circuit $\mathcal{A}=\left\{a_{1}, \ldots, a_{n+2}\right\}$ of cardinality $n+2$.

Output: A true declaration as to whether $Z_{+}(f)$ is empty or not, where $f(x):=\sum_{i=1}^{n+2} c_{i} x^{a_{i}}$.

Description:

1. If all the $c_{i}$ have the same sign then output " $Z_{+}(f)=\emptyset "$ and STOP.

2. Let $b=\left(b_{1}, \ldots, b_{n+2}\right) \in \mathbb{Z}^{n}$ be the vector obtained by applying Lemma 2.13 to $\mathcal{A}$. If $b$ or $-b$ has a unique negative coordinate $b_{j^{\prime}}$, and $c_{j^{\prime}}$ is the unique negative coordinate of $c$ or $-c$, then do the following:

(a) Replace $b$ by $-\operatorname{sign}\left(b_{j^{\prime}}\right) b$, replace $c$ by $-\operatorname{sign}\left(c_{j^{\prime}}\right) c$, and then reorder $b, c$, and $\mathcal{A}$ by the same permutation so that $b_{j^{\prime}}<0$ and $\left[b_{i}>0\right.$ iff $\left.i<j^{\prime}\right]$. 
(b) If $j^{\prime}<n+2$ and

$$
b_{j^{\prime}}^{-b_{j^{\prime}}} \prod_{i=1}^{j-1} c_{i}^{b_{i}}=c_{j^{\prime}}^{-b_{j^{\prime}}} \prod_{i=1}^{n+1} b_{i}^{b_{i}}
$$

then output " $Z_{+}(f)=\emptyset$ " and STOP.

(c) Decide via Algorithm 2.16 whether

$b_{j^{\prime}}^{-b_{j^{\prime}}} \prod_{i=1}^{j^{\prime}-1} c_{i}^{b_{i}} \stackrel{?}{>} c_{j^{\prime}}^{-b_{j^{\prime}}} \prod_{i=1}^{n+1} b_{i}^{b_{i}}$.

If so, output " $Z_{+}(f)=\emptyset "$ and STOP.

3. Output " $Z_{+}(f)$ is non-empty!" and STOP.

The correctness of Algorithm 3.2 follows directly from Theorems 2.19 and 2.18. In particular, note that $b_{i}$ is simply the signed volume of $\operatorname{Conv}\left(\mathcal{A} \backslash\left\{a_{i}\right\}\right)$. So the geometric interpretation $b$ or $-b$ having a unique negative coordinate is that the convex hull of the unique non-degenerate subcircuit of $\mathcal{A}$ is a simplex, with $a_{j^{\prime}}$ lying in its relative interior. Similarly, the geometric interpretation of $j^{\prime}<n+2$ is that $\mathcal{A}$ is a degenerate circuit. Finally, the product comparisons from Steps (b) and (c) simply decide the product inequalities stated in Theorem 2.18 and Theorem 2.19

So now we need only bound complexity, and this follows immediately from Lemma 2.17 (assuming we use Algorithm 2.15 for Step (b)).

It is worth noting that we need to compute the sign of a linear combination of logarithms only when the unique nondegenerate subcircuit $\mathcal{B}$ of $\mathcal{A}$ is a simplex, and all "vertex" coefficients have sign opposite from the "internal" coefficient. Also, just as in Remark 3.1 checking whether a given $f \in$ $\mathcal{F}_{n, n+2}$ lies in $\mathcal{F}_{n, n+2}^{*}$ can be done within $\mathbf{N} \mathbf{C}^{2}$ by computing $d=\operatorname{dim} \operatorname{Supp}(f)$ efficiently. Moreover, from our preceding proof, we see that deciding whether a circuit is degenerate (and extracting $\mathcal{B}$ from $\mathcal{A}$ when $\mathcal{A}$ is degenerate) can be done in $\mathbf{N C}^{2}$ as well, since we can set $\beta=1$ if we only want the signs of $\left(b_{1}, \ldots, b_{n+2}\right)$.

\section{Acknowledgements}

The authors thank Francisco Santos for earlier discussions on counting regular triangulations, and Frank Sottile for inviting the second author to an April 2008 meeting at the Institute Henri Poincare where a version of these results was presented. Thanks also to Dima Pasechnik for discussions, and Sue Geller and Bruce Reznick for detailed commentary, on earlier versions of this work. We also thank AIM and IMA for their hospitality and support while this paper was nearing completion at respective workshops on Random Analytic Surfaces and Complexity, Coding, and Communication. Finally, we thank Philippe Pébay and David C. Thompson for their great hospitality at Sandia National Laboratories where this paper was completed.

\section{REFERENCES}

[AM09] Avendaño, Martin and Mortari, Daniele, "The Multi-Impulse Orbit Transfer Problem," preprint,

Texas A\&M University, 2009.

[AR09] Avendaño, Martin and Rojas, J. Maurice, " $A$ Critical Radius for Low Complexity," in preparation, 2009.

[BS96] Bach, Eric and Shallit, Jeff, Algorithmic

Number Theory, Vol. I: Efficient Algorithms, MIT

Press, Cambridge, MA, 1996.

[Bak77] Baker, Alan, "The Theory of Linear Forms in Logarithms," in Transcendence Theory: Advances and
Applications: proceedings of a conference held at the University of Cambridge, Cambridge, Jan.-Feb., 1976, Academic Press, London, 1977.

[BHPR09] Bastani, Osbert; Hillar, Chris; Popov, Dimitar; and Rojas, J. Maurice, "Sums of Squares, Randomization, and Sparse Polynomials," in preparation, 2009.

[BG-V03] Basu, Saugata and Gonzalez-Vega, Laureano, Algorithmic and Quantitative Real Algebraic Geometry, Papers from the DIMACS Workshop on Algorithmic and Quantitative Aspects of Real Algebraic Geometry in Mathematics and Computer Science held at Rutgers University, Piscataway, NJ (March 12-16, 2001), DIMACS Series in Discrete Mathematics and Theoretical Computer Science, 60. [BPR06] Basu, Saugata; Pollack, Ricky; and Roy, Marie-Francoise, Algorithms in Real Algebraic Geometry, Algorithms and Computation in Mathematics, vol. 10, Springer-Verlag, 2006. [Ber03] Bernstein, Daniel J., "Computing Logarithm Intervals with the Arithmetic-Geometric Mean Iterations," available from http://cr.yp.to/papers.html .

[Ble04] Blekherman, Grigoriy, "Convexity properties of the cone of nonnegative polynomials," Discrete Comput. Geom. 32 (2004), no. 3, pp. 345-371. [Can88] Canny, John F., "Some Algebraic and Geometric Computations in PSPACE, "Proc. 20hㅡㄹ ACM Symp. Theory of Computing, Chicago (1988), ACM Press.

[Csa76] Csanky, L., "Fast Parallel Matrix Inversion Algorithms," SIAM J. Comput. 5 (1976), no. 4, pp. 618-623.

[DRRS07] Dickenstein, Alicia; Rojas, J. Maurice; Rusek, Korben; and Shih, Justin, " $\mathcal{A}$-Discriminants and Extremal Real Algebraic Geometry," Moscow Mathematical Journal, vol. 7, no. 3, (July-September, 2007).

[DL79] Dobkin, David and Lipton, Richard, "On the Complexity of Computations Under Varying Sets of Primitives, " J. of Computer and System Sciences 18, pp. 86-91, 1979.

[vdD86] van den Dries, Lou, "A generalization of the Tarski-Seidenberg theorem, and some nondefinability results," Bull. Amer. Math. Soc. (N.S.) 15 (1986), no. 2, pp. 189-193.

[vdD98] van den Dries, Lou, Tame topology and o-minimal structures, London Mathematical Society Lecture Note Series, 248, Cambridge University Press, Cambridge, 1998.

[EMP06] Algebraic geometry and geometric modeling, papers from a workshop held at University of Nice-Sophia Antipolis, Nice, Sept. 27-29, 2004, (edited by Mohamed Elkadi, Bernard Mourrain and Ragni Piene), Mathematics and Visualization,

Springer-Verlag, Berlin, 2006.

[GV04] Gabrielov, Andrei and Vorobjov, Nicolai, "Complexity of computations with Pfaffian and

Noetherian functions," Normal Forms, Bifurcations and Finiteness Problems in Differential Equations, pp. 211-250, Kluwer, 2004.

[GJ79] Garey, Michael R. and Johnson, David S. 
Computers and Intractability: A Guide to the Theory of NP-Completeness, A Series of Books in the Mathematical Sciences, W. H. Freeman and Co., San Francisco, Calif., 1979, x+338 pp.

[GKZ94] Gel'fand, Israel Moseyevitch; Kapranov, Misha M.; and Zelevinsky, Andrei V.; Discriminants, Resultants and Multidimensional Determinants, Birkhäuser, Boston, 1994.

[HLP88] Hardy, G. H.; Littlewood, J. E.; Pólya, G., Inequalities, reprint of the 1952 edition, Cambridge Mathematical Library, Cambridge University Press, Cambridge, 1988.

[Hir94] Hirsch, Morris, Differential Topology, corrected reprint of the 1976 original, Graduate Texts in Mathematics, 33, Springer-Verlag, New York, 1994.

[IRR07] Ibrahim, Ashraf; Rojas, J. Maurice; and Rusek, Korben, "First Steps in Algorithmic Arithmetic Fewnomial Theory," Math ArXiV preprint 0711.2562 . [Ili89] Iliopoulos, Costas S., "Worst Case Complexity Bounds on Algorithms for Computing the Canonical Structure of Finite Abelian Groups and the Hermite and Smith Normal Forms of an Integer Matrix," SIAM Journal on Computing, 18 (1989), no. 4, pp. 658-669. [KS99] Karpinski, Marek and Shparlinski, Igor, "On the computational hardness of testing square-freeness of sparse polynomials," Applied algebra, algebraic algorithms and error-correcting codes (Honolulu, HI, 1999), pp. 492-497, Lecture Notes in Comput. Sci., 1719, Springer, Berlin, 1999.

[Kho91] Khovanski, Askold, Fewnomials, AMS Press, Providence, Rhode Island, 1991.

[Las07] Lassere, Jean B., "A Sum of Squares

Approximation of Nonnegative Polynomials,"SIAM

Review, Vol. 49, No. 4, pp. 651-669.

[LM01] Lickteig, Thomas and Roy, Marie-Francoise, "Sylvester-Habicht Sequences and Fast Cauchy Index Computation," J. Symbolic Computation (2001) 31, pp. 315-341.

[LRW03] Li, Tien-Yien; Rojas, J. Maurice; and Wang, Xiaoshen, "Counting Real Connected Components of

Trinomial Curve Intersections and $m$-nomial

Hypersurfaces," Discrete and Computational

Geometry, 30 (2003), no. 3, pp. 379-414.

[Mig92] Mignotte, Maurice, Mathematics for Computer

Algebra, translated from the French by Catherine

Mignotte, Springer-Verlag, New York, 1992.

[Nes03] Nesterenko, Yuri, "Linear forms in logarithms of rational numbers," Diophantine approximation (Cetraro, 2000), pp. 53-106, Lecture Notes in Math., 1819, Springer, Berlin, 2003.

[Pap95] Papadimitriou, Christos H., Computational Complexity, Addison-Wesley, 1995.

[Par03] Parrilo, Pablo A., "Semidefinite programming relaxations for semialgebraic problems," Algebraic and geometric methods in discrete optimization, Math. Program. 96 (2003), no. 2, Ser. B, pp. 293-320. [PRT09] Pébay, Philippe; Rojas, J. Maurice; and Thompson, David C., "NP $\mathbf{P}_{\mathbb{R}}$-Completeness and Sparse Polynomials," in preparation, 2009.

[RY05] Rojas, J. Maurice and Ye, Yinyu, "On Solving Sparse Polynomials in Logarithmic Time," Journal of
Complexity, special issue for the 2002 Foundations of Computation Mathematics (FOCM) meeting, February 2005, pp. 87-110.

[RS09] Rojas, J. Maurice and Sethuraman,

Swaminathan, "Refined Asymptotics for Sparse Sums of Squares," extended abstract, submitted for publication. Also available as Math Arxiv preprint 0901.3786 .

[Sch00] Schmid, Joachim, "On the Complexity of the Real Nullstellensatz in the 0-Dimensional Case," J.

Pure Appl. Algebra 151 (2000), no. 3, pp. 301-308. [Sma87] Smale, Steve, "On the Topology of Algorithms I, ” Journal of Complexity 3 (1987), no. 2, pp. 81-89. [Sto98] Storjohann, Arne, "Computing Hermite and Smith normal forms of triangular integer matrices," Linear Algebra Appl. 282 (1998), no. 1-3, pp. 25-45. [Ste74] Stengle, G., "A nullstellensatz and a positivstellensatz in semialgebraic geometry," Math. Ann. 207 (1974) pp. 87-97.

[Sus79] Sussmann, Héctor J., "Subanalytic sets and feedback control," J. Differential Equations 31 (1979), no. 1, pp. 31-52.

[Tar51] Tarski, Alfred, A Decision Method for Elementary Algebra and Geometry, prepared for publication by J. C. C. McKinsey, University of California Press, Berkeley and Los Angeles, California, 1951.

\section{Appendix: Complexity, Viro Diagrams, and Post- poned Proofs \\ 4.1 Complexity Classes and 3CNFSAT}

A complete and rigourous description of the complexity classes we used can be found in Pap95. So for the convenience of the reader, we briefly review the following definitions:

$\mathbf{N C}^{i}$ The family of functions computable by Boolean circuit: with size polynomial in the input size and depth $O\left(\log ^{i}\right.$ InputSize $)$.

$\mathbf{P}$ The family of decision problems that can be done within time polynomial in the input size.

NP The family of decision problems where a "Yes" answer can be verified within time polynomial in the input size.

PSPACE The family of decision problems solvable within polynomial-time, provided a number of processors exponential in the input size is allowed.

DEFINITION 4.1. Recall that 3CNFSAT is the problem of deciding whether an n-variate Boolean formula of the form $B(X)=C_{1}(X) \wedge \cdots \wedge C_{N}(X)$ has a satisfying assignment, where each clause $C_{\ell}$ is of one of the following forms:

$X_{i} \vee X_{j} \vee X_{k}, \quad X_{i} \vee X_{j} \vee \neg X_{k}$,

$X_{i} \vee \neg X_{j} \vee \neg X_{k}, \neg X_{i} \vee \neg X_{j} \vee \neg X_{k}$,

$i, j, k \in[n]$ are pairwise distinct, $\left\lceil\frac{n}{3}\right\rceil \leq N \leq 8\left(\begin{array}{l}n \\ 3\end{array}\right)$, and a satisfying assigment consists of an assignment of values from $\{$ True, False $\}$ to the variables $X_{1}, \ldots, X_{n}$ yielding the equality $B(X)=$ True $\left[G J^{r} 79\right]$. We then define size $(B):=$

\footnotetext{
${ }^{4}$ This is the one time we will mention circuits in the sense of complexity theory: Everywhere else in this paper, our circuits will be combinatorial objects as in Definition 2.9.
} 
$3 N, a\left(x_{1}, x_{2}, x_{3}\right):=\left(x_{1}-2\right)\left(x_{2}-2\right)\left(x_{3}-2\right)$, and $b\left(x_{1}\right):=$ $\left(x_{1}-1\right)\left(x_{1}-2\right)$. Finally, to any 3CNFSAT clause $C_{\ell}$ as above, we associate a $4 \times 3$ polynomial system $H_{C_{\ell}}$ as follows: we respectively map clauses of the form $X_{i} \vee X_{j} \vee X_{k}$, $X_{i} \vee X_{j} \vee \neg X_{k}, X_{i} \vee \neg X_{j} \vee \neg X_{k}, \neg X_{i} \vee \neg X_{j} \vee \neg X_{k}$ to quadruples of the form

$$
\begin{gathered}
\left(a\left(x_{i}, x_{j}, x_{k}\right), b\left(x_{i}\right), b\left(x_{j}\right), b\left(x_{k}\right)\right), \\
\left(a\left(x_{i}, x_{j}, 3-x_{k}\right), b\left(x_{i}\right), b\left(x_{j}\right), b\left(x_{k}\right)\right), \\
\left(a\left(x_{i}, 3-x_{j}, 3-x_{k}\right), b\left(x_{i}\right), b\left(x_{j}\right), b\left(x_{k}\right)\right), \\
\left(a\left(3-x_{i}, 3-x_{j}, 3-x_{k}\right), b\left(x_{i}\right), b\left(x_{j}\right), b\left(x_{k}\right)\right) ;
\end{gathered}
$$

and we associate to the 3CNFSAT instance $B(X)$ a $(8 N-n) \times(8 N-n)$ polynomial system with integral coefficients, $F_{B}$, defined to be

$\left(H_{C_{1}}, \ldots, H_{C_{N}},\left(u_{1}-1\right)\left(v_{1}-1\right), \ldots,\left(u_{4 N-n}-1\right)\left(v_{4 N-n}-1\right)\right)$. In particular, assigning True (resp. False) to $X_{i}$ will correspond to setting $x_{i}=2$ (resp. $\left.x_{i}=1\right)$. $\diamond$

Note that $F_{B}$ has a natural and well-defined zero set in $\left(\mathbb{P}_{\mathbb{C}}^{1}\right)^{8 N-n}$ since its Newton polytopes are either axes-parallel line segments or 3 -cubes, and we can multihomogenize with $8 N-n$ extra variables.

Proof of Proposition 2.2; Assertions (1) and (2) of Proposition 2.2 are elementary. In particular, the last $4 N-n$ polynomials of $F_{B}$ simply ensure that $F_{B}$ has enough variables so that it is square. Assertion (3) follows easily from the classical Arithmetic-Geometric Inequality HLP88, Sec. 2.5, pp. 16-18]. Assertion (4) follows easily upon observing the inequalities $k \leq\left[k^{1 / \varepsilon}\right]^{\varepsilon}=M^{\varepsilon}<(n+M)^{\varepsilon}$ and the fact that $\operatorname{Newt}\left(t_{M}\right)$ is $M$-dimensional.

\subsection{Digression on Viro Diagrams}

Let us recall an elegant result of Oleg Viro on the classification of certain real algebraic hypersurfaces. In what follows, we liberally paraphrase from Proposition 5.2 and Theorem 5.6 of [GKZ94, Ch. 5, pp. 378-393].

Definition 4.2. Given any finite point set $\mathcal{A} \subset \mathbb{R}^{n}$, let us call any function $\omega: \mathcal{A} \longrightarrow \mathbb{R}$ a lifting, denote by $\pi: \mathbb{R}^{n+1} \longrightarrow \mathbb{R}^{n}$ the natural projection which forgets the last coordinate, and let $\hat{\mathcal{A}}:=\{(a, \omega(a)) \mid a \in \mathcal{A}\}$. We then say that the polyhedral subdivision $\Sigma_{\omega}$ of $\mathcal{A}$ defined by $\left\{\pi(Q) \mid Q\right.$ a lower ${ }^{5}$ facet of $\operatorname{Conv} \hat{\mathcal{A}}$ of dimension $\left.\operatorname{dim} \mathrm{Aff} A\right\}$ is induced by the lifting $\omega$, and we call $\Sigma_{\omega}$ a triangulation induced by a lifting iff every cell of $\Sigma_{\omega}$ is a simplex. Finally, given any $f(x)=\sum_{a \in \mathcal{A}} c_{a} x^{a} \in \mathbb{Z}\left[x_{1}, \ldots, x_{n}\right]$, we define $f_{\omega, \varepsilon}(x):=\sum_{a \in \mathcal{A}} c_{a} \varepsilon^{\omega(a)} x^{a}$ to be the toric perturbation of $f$ (corresponding to the lifting $\omega$ ). $\diamond$

DeFinition 4.3. Following the notation above, suppose $\operatorname{dim} \operatorname{Aff} \mathcal{A}=n$ and $\mathcal{A}$ is equipped with a triangulation $\Sigma$ induced by a lifting and a function $s: \mathcal{A} \longrightarrow\{ \pm\}$ which we will call a distribution of signs for $\mathcal{A}$. We then locally define a piece-wise linear manifold - the Viro diagram $\mathcal{V}_{\mathcal{A}}(\Sigma, s)$ - in the following local manner: For any $n$-cell $C \in \Sigma$, let $L_{C}$ be the convex hull of the set of midpoints of edges of $C$ with vertices of opposite sign, and then define $\mathcal{V}_{\mathcal{A}}(\Sigma, s):=\bigcup_{C \text { an } n \text {-cell }} L_{C}$. When $\mathcal{A}=\operatorname{Supp}(f)$ and $s$ is the corresponding sequence of coefficient signs, then we also call $\mathcal{V}(f):=\mathcal{V}_{\mathcal{A}}(\Sigma, s)$ the Viro diagram of $f . \diamond$

\footnotetext{
${ }^{5}$ A lower facet is simply a facet which has an inner normal with positive last coordinate.
}

EXAMPLE 4.4. The following figure illustrates 6 circuits of cardinality 4, each equipped with a triangulation induced by a lifting, and a distribution of signs. The corresponding (possibly empty) Viro diagrams are drawn in thicker lines. $\diamond$
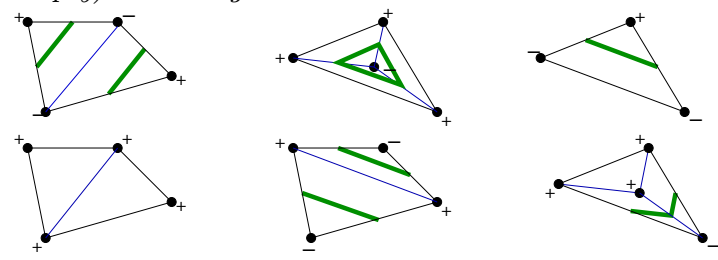

VIRO's ThEOREM. Suppose $f(x)=\sum_{a \in \mathcal{A}} c_{a} x^{a}$ is in $\mathbb{R}\left[x_{1}, \ldots, x_{n}\right]$ with $\operatorname{Supp}(f)=\mathcal{A}$ and $\operatorname{dim} \operatorname{Aff} \mathcal{A}=n, \omega$ is any lifting of $\mathcal{A}$, and define $s_{f}(a)=\operatorname{sign}\left(c_{a}\right)$ for all $a \in \mathcal{A}$. Then for any sufficiently small $\varepsilon>0, Z_{+}\left(f_{\omega, \varepsilon}\right)$ is isotopic to $\mathcal{V}_{\mathcal{A}}\left(\Sigma_{\omega}, s_{f}\right) \backslash \partial \operatorname{Conv} \mathcal{A}$. In particular, $\mathcal{V}_{\mathcal{A}}\left(\Sigma_{\omega}, s_{f}\right)$ is a disjoint finite union of piece-wise linear manifolds, each possibly having a non-empty boundary.

Lemma 4.5. Suppose $\mathcal{A}$ is a circuit, $\Sigma$ is a triangulation of $\mathcal{A}, n=\operatorname{dim} \operatorname{Aff} \mathcal{A}$, and $s$ is any distribution of signs on $\mathcal{A}$. Then $Z_{+}(f)$ smooth $\Longrightarrow Z_{+}(f)$ is isotopic to $\mathcal{V}_{\mathcal{A}}\left(\Sigma, s_{f}\right) \backslash$ $\partial \operatorname{Conv} \mathcal{A}$ for some $\Sigma$.

Proof of Lemma 4.5 By Lemma 2.13 it easily follows that $\mathcal{A}$ has at most 2 discriminant chambers in $\mathbb{R}_{+}^{n+2}$, and each such chamber contains a unique toric perturbation. Since the topology of $Z_{+}(f)$ is constant on any discriminant chamber containing $f$ by Lemma 2.12 , we are done.

Another important consequence of Viro's Theorem deals with how "roots at infinity" sometimes imply the existence of non-compact connected components for a positive zero set.

LEMMA 4.6. Assume $f$ has support such that every facet of $\operatorname{Newt}(f)$ is a simplex and coefficients such that $\mathcal{V}(f)$ intersects $\partial \mathrm{Newt}(f)$ for every underlying triangulation. Then $Z_{+}(f)$ has a non-compact connected component.

Employing our current notation, Lemma 4.6 follows directly from Lemma 15 of LRW03.

\subsection{More Postponed Proofs}

Proof of Lemma 2.5: Lemma 2.5 follows immediately from the following well-known factorization for integer matrices and its recent complexity bounds.

Definition 4.7. [Ili89, Sto98] Given any $M \in \mathbb{Z}^{m \times n}$, the Hermite factorization of $M$ is an identity of the form $U M=H$ where $U \in \mathbb{G L}_{m}$ and $H=\left[h_{i j}\right] \in \mathbb{Z}^{n \times n}$ is nonnegative and upper triangular, with all off-diagonal entries smaller than the positive diagonal entry in the same column. Finally, the Smith factorization of $M$ is an identity of the form $U M V=S$ where $U \in \mathbb{G L}_{m}, V \in \mathbb{G L}_{n}$, and $S=\left[s_{i j}\right] \in \mathbb{Z}^{m \times n}$ is diagonal, with $s_{i, i} \mid s_{i+1, i+1}$ for all $i$.

Lemma 4.8. [Ili89, Sto98] For any $M=\left[m_{i, j}\right] \in \mathbb{Z}^{n \times n}$, the Hermite and Smith factorizations of $M$ exist uniquely, and can be computed within $O\left(n^{4} \log ^{3}\left(n \max _{i, j}\left|m_{i, j}\right|\right)\right)$ bit operations. Furthermore, in the notation of Definition 4.7, the entries of $U, V, S$, and $H$ all have bit size $O\left(n^{3} \log ^{2}\left(2 n+\max _{i, j}\left|m_{i, j}\right|\right)\right)$. 
Proof of Lemma 2.6: Computing our desired canonical form $\bar{f}$ boils down to reordering monomials, performing a monomial change of variables, and a rescaling.

First, let us replace $f$ by $\operatorname{sign}\left(c_{1}\right) f$ and reorder the terms of $f$ supported on $\left\{a_{2}, \ldots, a_{n+1}\right\}$ so that $c_{2}, \ldots, c_{\ell^{\prime}}$ (resp. $\left.c_{\ell^{\prime}+1}, \ldots, c_{n+1}\right)$ are positive (resp. negative), for some unique $\ell^{\prime} \in\{1, \ldots, n+1\}$. We then form the $n \times n$ matrix $B$ whose whose $i \underline{\text { th }}$ column is $a_{i+1}-a_{1}$, for $i \in\{1, \ldots, n\}$. If necessary, let us also swap the terms supported on $\left\{a_{2}, a_{3}\right\}$ (or $\left\{a_{n}, a_{n+1}\right\}$ ), before defining $B$, so that $\operatorname{det} B>0$ and the sign condition defining $\ell^{\prime}$ is still preserved. (If $n=1$ then we can simply reorder terms so that $a_{1}<a_{2}$ and define $B$ accordingly.)

Letting $\tilde{f}(x):=\frac{f\left(x^{B^{-1}}\right)}{x^{B^{-1} a_{1}}}$ we then obtain by Proposition 2.4 that $Z_{+}(f)$ and $Z_{+}(\tilde{f})$ are diffeotopic. Moreover, we clearly have that

$\tilde{f}(x):=c_{1}+c_{2} x_{1}+\cdots+c_{n+1} x_{n}$ (remember we have permuted the terms of $f$, and thus the $c_{i}$ as well) where $c_{1}>0$. So we can now define $\bar{f}(x)$ to be $\tilde{f}\left(\frac{x_{1}}{\left|c_{2}\right|}, \ldots, \frac{x_{n}}{\left|c_{n+1}\right|}\right)$, define $\gamma$ to be the constant term of $\bar{f}$, and set $\ell:=\ell^{\prime}-1$. It is then easily verified that the coefficients of $x_{1}, \ldots, x_{\ell}\left(\right.$ resp. $\left.x_{\ell+1}, \ldots, x_{n}\right)$ are all 1 (resp. -1 ).

Since $\bar{f}$ was defined by rescaling the variables of $\tilde{f}$, and such a scaling of variables can easily be parametrized so as to induce a diffeotopy from $Z_{+}(\bar{f})$ to $Z_{+}(\tilde{f})$, Assertion (2) follows. Assertion (1) holds trivially since we never altered the difference between the number of positive and negative coefficients. That $\ell$ is computable in $\mathbf{N C}^{1}$ follows from Lemma 4.8 we can compute $\ell$ simply by sorting, which clearly lies in $\mathbf{N C}^{1}$.

Proof of Corollary 2.7 .

Assertion (1): Employing the canonical form $\bar{f}(x)=\gamma+$ $x_{1}+\cdots+x_{\ell}-x_{\ell+1}-\cdots-x_{n}$ of Lemma 2.6 (with $\gamma>0$ and $\ell \in\{0, \ldots, n\}$, by construction), the desired equivalence will follow upon proving that $\bar{f}$ has a root in $\mathbb{R}_{+}^{n} \Longleftrightarrow \ell<n$. The latter equivalence is trivially true. By Lemma 2.6 once more, the statement on diffeotopy type can be reduced to the special case of $\bar{f}$, which is also immediate.

Assertion (2): Dividing by a suitable monomial term, we can clearly assume that $a_{1}=\mathbf{O}$ and all the coefficients of $f$ are positive. So it suffices to prove that $f$ has a root in $\left(\mathbb{R}^{*}\right)^{n} \Longleftrightarrow$ there are indices $i \in[n]$ and $j \in\{2, \ldots, n+1\}$ with $a_{i, j}$ odd. Writing $f(x)=c_{1}+c_{2} x^{a_{2}}+\cdots+c_{n+1} x^{a_{n+1}}$, let us now prove the last equivalence.

$(\Longrightarrow)$ : To prove the contrapositive, assume every $a_{i, j}$ is even. Then the sign of $f$ is positive on all of $\left(\mathbb{R}^{*}\right)^{n}$ and $f$ thus has no roots in $\left(\mathbb{R}^{*}\right)^{n}$.

$(\Longleftarrow)$ : Reordering terms and variables, we can clearly assume that $a_{1,1}$ is odd. Letting $g\left(x_{1}\right):=f\left(x_{1}, 1, \ldots, 1\right)$, note that $g$ must be of the form $g\left(x_{1}\right)=c_{1}^{\prime}+c_{2}^{\prime} x^{a_{1,1}}$, where $c_{1}^{\prime}, c_{2}^{\prime}>0$. Since $g$ has the well-defined real root $-\sqrt[a_{1,1}]{\frac{c_{1}^{\prime}}{c_{2}^{\prime}}}, f$ then clearly has the root $\left(-\sqrt[a_{1}, 1]{\frac{c_{1}^{\prime}}{c_{2}^{\prime}}}, 1, \ldots, 1\right)$ which lies in $\left(\mathbb{R}^{*}\right)^{n}$.

Proof of Lemma 2.12; First, recall that subanalytic sets are those sets defined by projections of feasible sets of systems of analytic inequalities. In particular, $\mathcal{C}$ is a subanalytic set, and $\mathcal{C}$ is thus path connected since $\mathcal{C}$ admits a decom- position into connected cells. The existence of such a cell decomposition follows immediately from the $o$-minimality of subanalytic sets vdD98, vdD86. Moreover, $\mathcal{C}$ is path connected via differentiable paths by the classical density of $C^{\omega}$ functions among $C^{1}$ functions (see, e.g., Hir94, Ch. 2]).

So let $m=\# \mathcal{A}$ and let $\phi:=\left[\phi_{1}: \cdots: \phi_{m}\right]:[0,1] \longrightarrow \mathcal{C}$ be any differentiable path connecting $f$ and $g$. Also let $\mathcal{T}$ be the positive part of the real toric variety corresponding to $\operatorname{Conv}(\mathcal{A}), \mathcal{I}_{\mathcal{A}}:=\left\{(c, x) \in \mathbb{P}_{\mathbb{R}}^{m-1} \times \mathcal{T} \mid \sum_{i=1}^{m} c_{i} x^{a_{i}}=0\right\}$ the underlying (real) incidence manifold, and let $\pi$ denote the natural projection mapping $\mathbb{P}_{\mathbb{R}}^{m-1} \times \mathcal{T} \longrightarrow \mathbb{P}_{\mathbb{R}}^{m-1}$. Note then that $\phi$ induces an embedded smooth compact submanifold $M \subseteq I_{\mathcal{A}}$, consisting of all those $(c, x)$ with $c=\phi(t)$ and $\sum_{i=1}^{m} c_{i} x^{a_{i}}=0$ for some $t$. In particular, we see that $M$ is fibered over $[0,1]$ and that $\psi=\phi^{-1} \circ \pi$ is a Morse function on $M$ with no critical points in $[0,1]$. More to the point, we obtain a natural flow on $M$ inducing a diffeotopy $\delta$ between the zero sets of $f$ and $g$ in $\mathcal{T}$ [Hir94, Thm. 2.2, pg. 153].

To conclude, we simply observe that the intersection of $\psi^{-1}(t)$ with toric infinity is smooth for all $t \in[0,1]$ (by our assumption on the facets of $\operatorname{Conv}(\mathcal{A})$ ) and thus $\delta$ restricts to a diffeotopy between $Z_{+}(f)$ and $Z_{+}(g)$.

Proof of Lemma 2.13, With the exception of the assertion on complexity, Lemma 2.13 follows directly from GKZ94, Prop. 1.8, Pg. 274], GKZ94, Prop. 1.2, pg. 217], and the discussion following up to the end of Section B on page 218 of GKZ94. In particular, the factor $\beta$ takes into account that $\mathcal{A}$ may not affinely generate $\mathbb{Z}^{n}$, but is always the integral affine image of an $\mathcal{A}^{\prime}$ that is. So the sign condition arises simply from a binomial system (with odd determinant) that $\zeta$ must satisfy.

The assertion on the complexity of computing $\left(b_{1}, \ldots, b_{n+2}\right)$ follows immediately from Csanky's famous parallel algorithm for the determinant Csa76, combined with Lemma 4.8. Indeed, were it not for the gcd computation for $\beta$, we could instead assert an $\mathbf{N C}^{2}$ complexity bound.

Proof of Lemma 2.17; We first recall the following theorem:

Theorem 4.9. [BS96, Thm. 4.8.7, Sec. 4.8] Following the notation of Definition 2.14 there is a gcd-free basis for $\left\{\alpha_{1}, \ldots, \alpha_{N}\right\}$, with $\eta$, size $\left(\gamma_{i}\right)$, and $\operatorname{size}\left(e_{i j}\right)$ each polynomial in $\sum_{\ell=1}^{N} \operatorname{size}\left(\alpha_{\ell}\right)$, for all $i$ and $j$. Moreover, one can always find such a gcd-free basis using just $O\left(\left(\sum_{\ell=1}^{N} \operatorname{size}\left(\alpha_{\ell}\right)\right)^{2}\right)$ bit operations.

Returning to the proof of Lemma 2.17, note then that Algorithm 2.15] is correct and runs in the time stated by Theorem 4.9 and the naive complexity bounds for integer multiplication.

To prove the remaining half of our lemma, observe that the sign of $\alpha_{1}^{u_{1}} \cdots \alpha_{M}^{u_{M}}-\beta_{1}^{v_{1}} \cdots \beta_{N}^{v_{N}}$ is the same as the sign of $S:=\left(\sum_{i=1}^{M} u_{i} \log \alpha_{i}\right)-\left(\sum_{i=1}^{N} v_{i} \log \beta_{i}\right)$. Clearly then, $\left|S-\left[\left(\sum_{i=1}^{M} u_{i} A_{i}\right)-\left(\sum_{i=1}^{N} v_{i} B_{i}\right)\right]\right|<E / 2$ by the Nesterenko-Matveev Theorem. So Step (3) of Algorithm 2.16 indeed computes the sign of $S$ and we thus obtain correctness.

To see that Algorithm 2.16 runs within the time stated, first note that the algorithm computes $M$ (resp. $N$ ) approximations of logs of positive integers, each of size $O\left(\max _{i} \log \left|\alpha_{i}\right|\right)$ 
(resp. $\left.O\left(\max _{i} \log \left|\beta_{i}\right|\right)\right)$, correct in their first $O(E+\log M)$ (resp. $O(E+\log N)$ ) leading bits. Employing the explicit bit complexity estimates for fast multiplication of BS96. Table 3.1 , pg. 43], it is easily checked that Bernstein's method quoted above uses $O\left(b \log ^{2}(b) \log \log b\right)$ bit operations. So, via our chosen method for approximating logarithms [Ber03], we see that the complexity of Algorithm 2.16 is

$O\left(M(E+\log M) \log ^{2}(E+\log M) \log \log (E+\log M)\right.$ $\left.+N(E+\log N) \log ^{2}(E+\log N) \log \log (E+\log N)\right)$ $=O\left((M+N) E \log ^{2}(E) \log \log E\right)$. Upon observing that $M+N=O\left(\left(\sum_{i=1}^{M} \log \log \left|\alpha_{i}\right|\right)+\left(\sum_{i=1}^{N} \log \log \left|\beta_{i}\right|\right)\right)$, $(2 e)^{2}<30$, and $a b c \log (a b c) \leq a \log (a) b \log (b) c \log (c)$ for $a, b, c$ sufficiently large, our final asserted complexity bound follows easily.

Proof of Theorem 2.18; First note that Condition (1) implies that $f$ maintains the same (non-zero) sign throughout $\mathbb{R}_{+}^{n}$. So Condition (1) trivially implies that $Z_{+}(f)=\emptyset$, and we may assume henceforth that not all the coefficients of $f$ have the same sign.

Now, if Newt $(f)$ is not a simplex, then every point of $\mathcal{A}$ is a vertex of Newt $(f)$ and thus, independent of the triangulation, any Viro diagram for $\mathcal{A}$ must be non-empty. Since there are only two discriminant chambers (by Lemma 2.13), Lemma 4.5 thus implies that $Z_{+}(f)$ must be non-empty, assuming $\Delta_{\mathcal{A}}(f) \neq 0$. Lemma 2.13 tells us that $Z_{+}(f)$ must be non-empty if $\Delta_{\mathcal{A}}(f)=0$. So we may assume henceforth that $\operatorname{Newt}(f)$ is a simplex and that $\Delta_{\mathcal{A}}(f) \neq 0$.

Continuing our focus on Condition (2), note that if the sign equalities from Condition (2) fail, then there must exist coefficients $c_{i}$ and $c_{i^{\prime}}$ of opposite sign such that $a_{i}$ and $a_{i^{\prime}}$ vertices of $\operatorname{Newt}(f)$. So, again, independent of the triangulation, any Viro diagram for $\mathcal{A}$ must be non-empty and thus (just as in the preceding paragraph) $Z_{+}(f)$ must again be non-empty. So we may assume henceforth that the sign equalities from Condition (2) hold.

At this point, it is clear that we need only show that (under our current assumptions) $Z_{+}(f)=\emptyset \Longleftrightarrow$ the discriminant inequality from Condition (2) holds. Toward this end, observe that the lifting that assigns $a_{j^{\prime}} \mapsto-1$ and $a_{i} \mapsto 0$ for all $i \neq j^{\prime}$ induces the unique triangulation of $\mathcal{A}$ consisting of a single simplex. In particular, the underlying Viro diagram is empty, due to the sign equalities. So by Viro's Theorem and Lemma 4.5, $Z_{+}(f)$ is empty for $\left|c_{j^{\prime}}\right|$ sufficiently small. By Lemmata 2.12 and 2.13, this topology persists for $\left|c_{j^{\prime}}\right|$ just small enough to enforce the discriminant sign stated in Condition (2), so we are done.

Proof of Theorem 2.19; First, we observe that via an argument almost identical to the proof of Lemma 2.6 we can find a monomial change of variables (and multiply by a suitable monomial term) so that $\bar{f}(x):=x^{v} f\left(x^{M}\right)$ is of the form $c_{1}+c_{2} x_{1}^{u_{1}}+\cdots+c_{j^{\prime}-1} x_{j^{\prime}-2}^{u_{j^{\prime}-2}}+c_{j^{\prime}} x^{\alpha}+c_{j^{\prime}+1} x_{j^{\prime}-1}^{u_{j^{\prime}-1}}+\cdots+c_{n+2} x_{n}^{u_{n}}$, where $u_{1}, \ldots, u_{n} \in \mathbb{N}$ and $\alpha \in \mathbb{N}^{j^{\prime}-2} \times\{0\}^{n-j^{\prime}+2}$. In particular, defining $\bar{f}_{\mathcal{B}}(x)=c_{1}+c_{2} x_{1}^{u_{1}}+\cdots+c_{j^{\prime}-1} x_{j^{\prime}-2}^{u_{j^{\prime}-2}}+c_{j^{\prime}} x^{\alpha}$, it is clear that $Z_{+}(\bar{f})$ is nothing more than the intersection of the graph of $\bar{f}_{\mathcal{B}}$ (which is analytic on $\mathbb{R}_{+}^{j^{\prime}-2}$ ) with an orthant. So $Z_{+}(\bar{f})$ is smooth and thus, by Proposition 2.4, we obtain that $Z_{+}(f)$ is diffeotopic to $Z_{+}(\bar{f})$. It thus suffices to prove our lemma for $\bar{f}$. Note also that the conditions on $Z_{+}(f)$ allegedly characterizing $Z_{+}(f)=\emptyset$ are preserved under monomial multiples and monomial changes of variables.
Observe now that if $Z_{+}\left(\bar{f}_{\mathcal{B}}\right)$ has $\geq 2$ points then $Z_{+}\left(\bar{f}_{\mathcal{B}}\right)$ must contain a smooth point $\zeta$, thanks to Lemma 2.13. So then, by the implicit function theorem, $\bar{f}_{\mathcal{B}}$ must attain both positive and negative values in a neighborhood of $\zeta \in \mathbb{R}_{+}^{j}$. Since the range of $\bar{f}_{B^{\prime}}:=c_{j^{\prime}+1} x_{j^{\prime}-1}^{u_{j^{\prime}-1}}+\cdots+c_{n+2} x_{n}^{u_{n}}$ (over $\mathbb{R}^{n-j^{\prime}+2}$ ) must contain the positive ray or the negative ray, and $\bar{f}=\bar{f}_{\mathcal{B}}-\left(-\bar{f}_{\mathcal{B}^{\prime}}\right)$, we thus obtain that $Z_{+}(\bar{f})$ is nonempty. So we may assume henceforth that $\# Z_{+}\left(\bar{f}_{\mathcal{B}}\right) \leq 1$.

To further simplify matters, observe that if all the coefficients of $\bar{f}_{\mathcal{B}}$ have the same sign then, by assumption, we must have a coefficient of $\bar{f}_{\mathcal{B}^{\prime}}$ differing in sign from $c_{1}$. So then, $\bar{f}_{\mathcal{B}}\left(\mathbb{R}_{+}^{j^{\prime}-2}\right)$ has constant sign and $-\bar{f}_{\mathcal{B}^{\prime}}\left(\mathbb{R}^{n-j^{\prime}+2}\right)$ contains a ray of the same sign. Therefore $Z_{+}(\bar{f})$ is non-empty. We may therefore also assume that not all the coefficients of $\bar{f}_{\mathcal{B}}$ have the same sign.

We can therefore conclude by studying the two cases $Z_{+}\left(\bar{f}_{\mathcal{B}}\right)=\emptyset$ and $\# Z_{+}\left(\bar{f}_{\mathcal{B}}\right)=1$, combined with all our assumptions so far.

$\left(\boldsymbol{Z}_{+}\left(\bar{f}_{\mathcal{B}}\right)=\emptyset\right)$ : By Theorem 2.18 applied to $\bar{f}_{\mathcal{B}}$, we see that Conditions (a) and (b) must hold (and the inequality in (b) strictly so), with the possible exception of the equalities $-\operatorname{sign}\left(c_{j^{\prime}}\right)=\operatorname{sign}\left(c_{i}\right)$ for all $i>j^{\prime}$. So we need only show that $Z_{+}(\bar{f})=\emptyset \Longleftrightarrow-\operatorname{sign}\left(c_{j^{\prime}}\right)=\operatorname{sign}\left(c_{i}\right)$ for all $i>j^{\prime}$. By an argument almost identical to the last paragraph, we can obtain that $\operatorname{sign}\left(c_{j^{\prime}}\right)=\operatorname{sign}\left(c_{i}\right)$ for some $i>j^{\prime} \Longleftrightarrow \bar{f}_{\mathcal{B}}\left(\mathbb{R}_{+}^{j^{\prime}}\right)$ and $-\bar{f}_{\mathcal{B}^{\prime}}\left(\mathbb{R}_{+}^{n-j^{\prime}+2}\right)$ intersect. So the only way we can have $Z_{+}(\bar{f})=\emptyset$ is for the equalities $-\operatorname{sign}\left(c_{j^{\prime}}\right)=\operatorname{sign}\left(c_{i}\right)$ to hold for all $i>j^{\prime}$.

$\left(\# Z_{+}\left(\bar{f}_{\mathcal{B}}\right)=1\right)$ : Clearly, the sole point of $Z_{+}\left(\bar{f}_{\mathcal{B}}\right)$ must be singular, and thus $\Delta_{\mathcal{A}}(f)=0$, which in turn enforces Condition (b) (with equality).

Now, if $\operatorname{Conv}(\mathcal{B})$ is not a simplex, then every one of its faces is a simplex, since $\mathcal{B}$ is a non-degenerate circuit. Moreover, all Viro diagrams of $\bar{f}_{\mathcal{B}}$ (regardless of triangulation) are non-empty and intersect $\partial \operatorname{Conv}(\mathcal{B})$. So, by Lemma 4.6. $Z_{+}\left(\bar{f}_{\mathcal{B}}\right)$ must contain an unbounded connected component and thus $\# \mathbb{Z}_{+}\left(\bar{f}_{\mathcal{B}}\right)>2$. So we may assume that $\operatorname{Conv}(\mathcal{B})$ is a simplex.

At this point, we need only prove that the sign equalities of Condition (a) must hold. Toward this end, note that if $\operatorname{sign}\left(c_{j^{\prime}}\right)=\operatorname{sign}\left(c_{i}\right)$ for some $i \in\left\{1, \ldots, j^{\prime}-1\right\}$ then all Viro diagrams of $\bar{f}_{\mathcal{B}}$ (regardless of triangulation) are nonempty and intersect $\partial \operatorname{Conv}(\mathcal{B})$. So, by Lemma 4.6 again, $Z_{+}\left(\bar{f}_{\mathcal{B}}\right)$ must contain an unbounded connected component forcing $\# \mathbb{Z}_{+}\left(\bar{f}_{\mathcal{B}}\right)>2$ again. We can therefore assume that $-\operatorname{sign}\left(c_{j^{\prime}}\right)=\operatorname{sign}\left(c_{i}\right)$ for all $i \in\left\{1, \ldots, j^{\prime}-1\right\}$.

To conclude, we will show that $-\operatorname{sign}\left(c_{j^{\prime}}\right) \bar{f}_{\mathcal{B}}$ attains only nonnegative values on $\mathbb{R}_{+}^{j^{\prime}-2}$. (This will enforce our final desired sign equalities $-\operatorname{sign}\left(c_{j^{\prime}}\right)=\operatorname{sign}\left(c_{i}\right)$ for all $i>j^{\prime}$ - simply by comparing the range of $\bar{f}_{\mathcal{B}}$ and $\bar{f}_{\mathcal{B}^{\prime}}$ just as before.) First note that $\bar{f}_{\mathcal{B}}\left(\mathbb{R}_{+}^{j^{\prime}-2}\right)$ is unaffected by invertible monomial changes or positive scalings of variables (thanks to Proposition 2.4). Also, note that $\operatorname{sign}\left(\bar{f}_{\mathcal{B}}(x)\right)=$ $\operatorname{sign}\left(x^{v} \bar{f}_{\mathcal{B}}(x)\right)$ for any $x \in \mathbb{R}_{+}^{j^{\prime}-2}$ and $v \in \mathbb{R}^{j^{\prime}-2}$. So it clearly suffices to show that $g(x):=1+x_{1}+\cdots+x_{j^{\prime}-2}-\gamma x^{\alpha}$ attains only nonnegative values, where now $\alpha \in \mathbb{R}_{+}^{j^{\prime}-2}$ and $\alpha_{1}+\cdots+\alpha_{j^{\prime}-2}<1$ (since $a_{j^{\prime}}$ lies in the interior of $\operatorname{Newt}\left(\bar{f}_{\mathcal{B}}\right)$ ), and $\gamma=\frac{\left(1-\alpha_{1}-\cdots-\alpha_{j^{\prime}-2}\right)^{\alpha_{1}+\cdots+\alpha_{j^{\prime}-2}}}{\left(1-\alpha_{1}-\cdots-\alpha_{j^{\prime}-2}\right) \alpha_{1}^{\alpha_{1} \cdots \alpha_{j^{\prime}-2}}}$ (since Condition (b) 
holds with equality). That $g$ attains only nonnegative values is then clearly equivalent to the inequality

$$
1+x_{1}+\cdots+x_{j^{\prime}-2} \geq \gamma x^{\alpha}
$$

which is in turn equivalent to

$\left(1-\alpha_{1}-\cdots-\alpha_{j^{\prime}-2}\right)\left(1+x_{1}+\cdots+x_{j^{\prime}-2}\right)$
$\quad \geq \prod_{i=1}^{j^{\prime}-2}\left(\frac{\left(1-\alpha_{1}-\cdots-\alpha_{j^{\prime}-2}\right) x}{\alpha_{i}}\right)^{a_{i}}$

$\left(1-\alpha_{1}-\cdots-\alpha_{j^{\prime}-2}\right)+\alpha_{1} u_{1}+\cdots+\alpha_{j^{\prime}-2} u_{j^{\prime}-2} \geq \prod_{i=1}^{j^{\prime}-2} u_{i}^{\alpha_{i}}$

upon substituting $x_{i}=\alpha_{i} u_{i} /\left(1-\alpha_{1}-\cdots-\alpha_{j^{\prime}-2}\right)$. The last inequality is simply the weighted Arithmetic-Geometric Inequality HLP88, so we are done. 